\title{
Expedition 314 methods ${ }^{1}$
}

\author{
Expedition 314 Scientists $^{2}$
}

\section{Chapter contents}

Introduction $\ldots \ldots \ldots \ldots \ldots \ldots \ldots \ldots 1$ Logging while drilling . . . . . . . . . . 1 Onboard data flow and quality check .....6 6 Log characterization and lithologic interpretation ............. 7 Physical properties .............. 8 Structural geology and geomechanics . . . . .99 Log-seismic correlation . . . . . . . . . . . 11 References.................... 13 Figures.................. 15 Tables.....................29

${ }^{1}$ Expedition 314 Scientists, 2009. Expedition 314 methods. In Kinoshita, M., Tobin, H., Ashi, J., Kimura, G., Lallemant, S., Screaton, E.J., Curewitz, D., Masago, H., Moe, K.T., and the Expedition 314/315/316 Scientists, Proc. IODP, 314/315/316: Washington, DC (Integrated Ocean Drilling Program Management International, Inc.). doi:10.2204/iodp.proc.314315316.112.2009 'Expedition 314/315/316 Scientists' addresses.

\section{Introduction}

Integrated Ocean Drilling Program (IODP) Expedition 314 is the first step in a multiexpedition, multiyear project to carry out the Nankai Trough Seismogenic Zone Experiment (NanTroSEIZE). The three expeditions that make up Stage 1 of the project focus on coring and logging operations at high-priority riserless sites on the Kumano transect. During Expedition 314, we focused exclusively on in situ measurements of subseafloor physical properties, lithology, stress, and geomechanics using logging-while-drilling (LWD) techniques, including real-time uphole data transmission (commonly referred to as measurement while drilling [MWD]). In this chapter, we explain the operation of LWD instruments and the physical principles behind the geophysical measurements obtained. In addition, we describe the methods used by shipboard scientists to arrive at the data analyses and interpretations reported in the site chapters of this volume.

\section{Logging while drilling}

During Expedition 314, six LWD and MWD tools were deployed under the contract by the Global Ocean Development Inc. with Schlumberger Drilling and Measurements Services. LWD surveys have been successfully conducted during previous Ocean Drilling Program (ODP) and IODP expeditions on the JOIDES Resolution with various tools of different generations, focusing on density, porosity, resistivity, gamma ray, and sonic velocity measurements. ODP Leg 196 was the last use of LWD in the Nankai Trough off Cape Muroto, $\sim 200 \mathrm{~km}$ southwest of the current locations (Mikada, Becker, Moore, Klaus, et al., 2002). During Expedition 314, the first operation of a ground-breaking complex drilling project with multiple expeditions over several stages, scientists conducted LWD at all NanTroSEIZE Stage 1 sites using the most advanced tools in scientific ocean drilling history.

LWD and MWD tools measure different parameters. LWD tools measure in situ formation properties with instruments that are located in special drill collars immediately above the drill bit. The LWD and MWD tools used during Expedition 314 include several of Schlumberger's VISION series tools, namely geoVISION, adnVISION, sonicVISION, and seismicVISION, in addition to MWD and annular-pressure-while-drilling (APWD) tools. Figure F1 shows the configuration of the LWD-MWD bottom-hole assembly (BHA), and 
the set of measurements recorded from LWD-MWD tools are listed in Tables T1, T2, and T3.

LWD measurements are made shortly after the hole is opened with the drill bit and before continued drilling operations adversely affect in situ properties and borehole stability. Fluid invasion into the borehole wall is also reduced relative to wireline logging because of the shorter elapsed time between drilling and taking measurements. MWD tools measure downhole drilling parameters (e.g., collar rotation) and annulus pressure and assure communication between tools. During drilling operations, these measurements are combined with surface rig floor parameters for easier drilling monitoring (e.g., weight on bit, torque, etc.) and quality control. The APWD sensor is included with the MWD sensors for safety monitoring and provides measurements of downhole pressure in the annulus, which are also converted to equivalent circulating density (ECD; density of the circulating drilling fluid when pumping). Downhole pressure and ECD are crucial parameters used to detect any inflow from the formation or obstruction (collapse of borehole walls), characterized by increases in APWD and ECD, or loss of circulation caused by permeable formations or faults, characterized by a decrease in APWD.

The key difference between LWD and MWD tools is that LWD data are recorded into downhole memory and retrieved when the tools reach the surface, whereas MWD data and a selection of LWD data are transmitted through the drilling fluid within the drill pipe by means of a modulated pressure wave (mud pulsing) at a rate of 6 bps (bits per second) and monitored in real time. The term LWD is often used more generically to cover both LWD and MWD type measurements, as the MWD tool is required during any LWD operation to provide communication between the LWD tools and the surface.

The LWD equipment is battery powered and uses erasable/programmable read-only memory chips to store the logging data until they are downloaded. The LWD tools take measurements at evenly spaced time intervals using a downhole clock installed in each tool and are synchronized with a depth tracking system on the rig that monitors time and drilling depth. After drilling, the LWD tools are retrieved and the data downloaded from each tool to a computer. Synchronization of the uphole and downhole clocks allows merging of the time-depth data (from the surface system) and the downhole time-measurement data (from the tools) into depth-measurement data files. The resulting depth-measurement data are transferred to the processing systems in the Downhole Data Processing Room for the Logging Staff Scientist and systematically distributed to the data serv- ers for the science party to interpret. Data flow is described in the next section.

\section{Systems and tools}

\section{Depth tracking systems}

LWD tools record data as a function of time. The Schlumberger integrated drilling and logging (IDEAL) surface system records the time and depth of the drill string below the rig floor. LWD operations aboard the $\mathrm{D} / \mathrm{V}$ Chikyu require accurate and precise depth tracking and the ability to independently measure and evaluate the position of the traveling block and top drive system in the derrick, heave of the vessel by the action of waves/swells and tides, and action of the motion compensator. The length of the drill string (combined length of the BHA and the drill pipe) and the position of the top drive in the derrick are used to determine the depth of the drill bit and rate of penetration. The system configuration is illustrated in Figure F2. A hook-load sensor is used to measure the weight of the load on the drill string and can be used to detect whether the drill string is in-slips or out-ofslips. When the drill string is in-slips (i.e., the top of the drill string is hung on the rig floor by the "slip" tool and is detached from the top drive) and motion from the blocks or motion compensator will not have any effect on the depth of the bit, the drawworks encoder information does not augment the recorded bit depth. It is clear when the drill string is out-of-slips (i.e., the drill string is connected to the top drive and is free from the rig floor). The heave of the ship will still continue to affect the bit depth whether the drill string is in-slips or out-of-slips.

The rig instrumentation system used by the drillers measures and records heave and the motion of the cylinder of the active compensator among many other parameters at the rig floor. The Chikyu uses a crown-mounted motion compensator (CMC) (Fig. F2), which is installed on the top of the derrick to reduce the influence of heave on the drill string and to raise the accuracy of the bit weight measurement. The CMC is united with the crown block, which is a stationary pulley, and absorbs tension by moving the crown block up and down according to the hull's up and down motion. When the crown block oscillates, the difference is absorbed by the change in the position of the horizontally overhung pulley even though the length of cable changes between the drawworks and the deadline anchor.

\section{Measurement-while-drilling (PowerPulse) and annulus pressure tools}

The MWD tool is the most basic but most important tool for operation data. This tool allows real-time 
two-way communication between LWD tools and the surface. MWD tools have previously been deployed during ODP Legs 188 (O'Brien, Cooper, Richter, et al., 2001), 196 (Mikada, Becker, Moore, Klaus, et al., 2002), and 204 (Tréhu, Bohrmann, Rack, Torres, et al., 2003) and IODP Expeditions 308 (Flemings, Behrmann, John, et al., 2006) and 311 (Riedel, Collett, Malone, et al., 2006). During Expedition 314, the Schlumberger MWD PowerPulse tool was used in combination with the APWD tool for pilot hole drilling and with LWD tools in other applications (Fig. F1).

MWD data are transmitted by means of a pressure wave through the fluid within the drill pipe (fluid pulse telemetry). The $63 / 4$ inch $(17 \mathrm{~cm})$ diameter MWD PowerPulse tool operates by generating a continuous mud-wave transmission within the drilling fluid and by changing the phase of this signal (frequency modulation) to convert relevant bit words representing information from various sensors (Fig. F3A). The data are compressed and coded digitally in pressure pulses that are sent up the well through the drilling fluid. Figure F3B illustrates the MWD fluid pulse telemetry system and a representative pressure wave. Drilling fluid pulses are recorded on two pressure transducers (signal pressure transducers) mounted on the standpipe manifold and the gooseneck of the standpipe where they are automatically decoded and uncompressed by the surface equipment. With the MWD fluid pulsing system, pulse rates range from 1 to 8-12 bps, depending primarily on water depth and fluid density. During Expedition 314 , pulse rates of 3 bps were achieved for MWDAPWD operations (pilot holes) and $6 \mathrm{bps}$ for the LWD holes.

The MWD parameters transmitted by fluid pulses include tool status information, vibrations, shocks, and tool stick-slip for continuous monitoring of the drilling operation. The latter measurements are made using paired strain gauges, accelerometers, and lateral shock sensors near the base of the MWD collar. A list of the main MWD parameters is given in Table T1. Tables T4 and T5 list typical telemetry frames sent in real time, showing measurements recorded using the MWD-APWD (PowerPulse) tools and their update rates. These data are transmitted to the surface. The comparison of MWD drilling parameters with rig instrumentation system data and ship heave information is used to improve drilling control and monitor any inflow or loss of circulation during drilling.

During LWD operations, the mud pulse system also transmitted a limited set of geophysical data from the adnVISION, geoVISION, sonicVISION, and seismicVISION LWD tools to the surface in real time.
These scientific measurements include gamma ray values, resistivity, bulk density, neutron porosity, compressional velocity ( $P$-wave), and seismic waveforms. Measurement parameters from each LWD collar were updated at rates corresponding to $15 \mathrm{~cm}$ to $1.5 \mathrm{~m}$ depth intervals, depending on the initialized values and rate of penetration (ROP) of the tool (Tables T4, T5). The combination of all these data confirmed the operational status of each tool and provided real-time logs for identifying lithologic contacts and potential shallow-water flow or overpressured zones. The real-time data also became the only source of information in cases when the data recorded in memory could not be retrieved (e.g., Site C0001 adnVISION data and all data for Site C0003).

\section{adnVISION tool}

The adnVISION tool is similar in principle to the older compensated density neutron tool (AnadrillSchlumberger, 1993; Moore, Klaus, et al., 1998). The density section of the tool uses a $1.7 \mathrm{Ci}{ }^{137} \mathrm{Cs}$ gamma ray source in conjunction with two gain-stabilized scintillation detectors to provide a borehole-compensated density measurement (Table T6). The detectors are located 5 and 12 inches (12.7 and 30.48 $\mathrm{cm}$ ) below the source (Fig. F4). The number of Compton scattering collisions (change in gamma ray energy by interaction with the formation electrons) is related to the formation density (Schlumberger, 1989).

Returns of low-energy gamma rays are converted to a photoelectric factor (PEF) value, measured in barns per electron. The PEF value depends on electron density and therefore responds to bulk density and lithology (Anadrill-Schlumberger, 1993). PEF value is also particularly sensitive to low-density, high-porosity zones.

The density source and detectors are positioned behind windows in the blade of $8 \frac{1}{4}$ inch $(25.9 \mathrm{~cm})$ integral blade stabilizer. This geometry forces the sensors against the borehole wall, thereby reducing the effects of borehole irregularities and drilling. Neutron logs are processed to eliminate the effects of borehole diameter, tool size, temperature, drilling mud hydrogen index (dependent on mud weight, pressure, and temperature), mud and formation salinities, lithology, and other environmental factors (Schlumberger, 1994). The vertical resolution of the density and photoelectric effect measurements is $\sim 6$ and 2 inches, respectively.

For measurement of tool standoff and estimated borehole size, a $670 \mathrm{kHz}$ ultrasonic caliper is available on the tool. The ultrasonic sensor is aligned with, and located just below, the density detectors. This sensor has an accuracy of \pm 0.1 inch and a verti- 
cal resolution of $\sim 6$ inches. In this position the sensor can also be used as a quality control for the density measurements.

Neutron porosity measurements are obtained using fast neutrons emitted from a $10 \mathrm{Ci}$ americium oxideberyllium (AmBe) source. Hydrogen quantities in the formation largely control the rate at which the neutrons slow down to epithermal and thermal energies. The energy of the detected neutrons has an epithermal component because much of the incoming thermal neutron flux is absorbed as it passes through the 1 inch drill collar. Neutrons are detected in near- and far-spacing detector banks, located 12 and 24 inches (30.48 and $60.96 \mathrm{~cm})$, respectively, above the source (Fig. F4). The vertical resolution of the tool under optimum conditions is $\sim 12$ inches $(34 \mathrm{~cm})$. The neutron logs are affected to some extent by the lithology of the matrix rock because the neutron porosity unit is calibrated for a water-saturated sandstone environment (Schlumberger, 1989).

The azimuthal measurement from the adnVISION tool is not reliable in wells with low deviation $\left(<10^{\circ}\right.$ inclination). In such environments, an average or maximum value should be used instead. Data output from the adnVISION tool include apparent neutron porosity (i.e., the tool does not distinguish between pore water and lattice-bound water), formation bulk density, and photoelectric factor. The density logs graphically presented here have been "rotationally processed" to show the average density that the tool reads while it is rotating. In addition, the adnVISION tool outputs an inferred density caliper record based on the standard deviation of density measurements made at high sampling rates around the circumference of the borehole. The measured standard deviation is compared with that of an in-gauge borehole and the difference is converted to the amount of borehole enlargement (Anadrill-Schlumberger, 1993). A standoff of $<1$ inch $(2.54 \mathrm{~cm})$ between the tool and the borehole wall indicates good borehole conditions, for which the density log values are considered to be accurate to $\pm 0.015 \mathrm{~g} / \mathrm{cm}^{3}$ (AnadrillSchlumberger, 1993).

Logging while drilling is a challenging environment for formation density measurement. The measurement is strongly affected by tool motion and influenced by the drilling fluid composition. The net effect is formation density and PEF measurements that can be inaccurate or misleading. The accuracy of the measurements can be greatly improved by acquiring data in both depth and azimuthal dimensions, assembling these data into a two-dimensional image, and selecting the density measurements least influenced by borehole effects from this image. In LWD tools, and in particular the adnVISION tool, azi- muthal data are acquired most economically from one set of sensors swept around the borehole by the rotation of the drill string.

The image-derived density algorithm uses the compensated density image to compute a single compensated density. It identifies which sectors at each depth level provide the highest quality measurements and computes a density measurement based on those sectors. It essentially automates what a skilled log analyst does when interpreting a density image. The algorithm consists of the following three steps:

1. Quality factor computation. For each depth level and sector, the short- and long-spaced densities and volumetric PEF are used to compute a quality factor. The quality factor is based on qualitative expectations and an empirical choice of parameters. Larger quality factors represent more accurate density measurements.

2. Toolpath identification. As a function of depth, the centroid of the region of high-quality measurements defines a toolpath. The toolpath can be thought of as the path of closest approach of the tool to the formation. This path is computed from the quality factor at each depth level by a partial Fourier decomposition.

3. Density calculation. The density is computed at each depth level by averaging the bulk density over four sectors centered on the toolpath. Fractional sectors are accounted for by linear interpolation. These steps are described in detail in Radkte et al. (2003).

By construction, this algorithm yields the highest quality density and PEF measurements possible. This technique has several other advantages: it is computed only from density sensor data, it is immune to the statistical bias and limited applicability of maximum density approaches, and the toolpath serves as a powerful quality control indicator.

\section{geoVISION tool}

The geoVISION resistivity tool is based on resistivityat-the-bit (RAB) technology, which was designed to provide real-time at-bit resistivity data. This explains why numerous geoVISION measurement acronyms include RAB in their name (see Table T3). The geoVISION resistivity tool provides resistivity measurements and electrical images of the borehole wall, calibrated in a homogeneous medium. In addition, the geoVISION tool contains a scintillation counter that provides a total gamma ray measurement (Fig. F5).

The geoVISION tool is connected directly above the drill bit and uses the lower portion of the tool and the bit as a measuring electrode. This allows the tool 
to provide a bit resistivity measurement with a vertical resolution just a few centimeters longer than the length of the bit. A $1 \frac{1 / 2}{2}$ inch $(4 \mathrm{~cm})$ electrode is located $102 \mathrm{~cm}$ from the bottom of the tool and provides a focused lateral resistivity measurement (ring resistivity) with a vertical resolution of 2-3 inches $(5-7.5 \mathrm{~cm})$. The characteristics of ring resistivity are independent of where the geoVISION tool is placed in the BHA, and its depth of investigation is $\sim 7$ inches $(17.8 \mathrm{~cm}$; diameter of investigation $\approx 22$ inches). In addition, button electrodes provide shallow-, medium-, and deep-focused resistivity measurements as well as azimuthally oriented images. These images can then reveal information about formation structure and lithologic contacts. The button electrodes are $\sim 1$ inch $(2.5 \mathrm{~cm})$ in diameter and reside on a clamp-on sleeve. The buttons are longitudinally spaced along the geoVISION tool to render staggered depths of investigation of $\sim 1,3$, and 5 inches $(2.5,7.6$, and $12.7 \mathrm{~cm})$. The spacing provides multiple depths of investigation for quantifying invasion profiles and fracture identification (drilling induced versus natural). Vertical resolution and depth of investigation for each resistivity measurement are shown in Table T7. For environmental correction of the resistivity measurements, drilling fluid resistivity and temperature are also measured (Schlumberger, 1989).

The tool's orientation system uses Earth's magnetic field as a reference to determine the tool position with respect to the borehole as the drill string rotates, thus allowing both azimuthal resistivity and gamma ray measurements. The gamma ray sensor has a range of operability of 0-250 gAPI and an accuracy of $\pm 7 \%$ corresponding to a statistical resolution of \pm 3 gAPI at $100 \mathrm{API}$ and ROP of $30 \mathrm{~m} / \mathrm{h}$. Its depth of investigation is between 5 and 15 inches. The azimuthal resistivity measurements are acquired with a $\sim 6^{\circ}$ resolution, whereas gamma ray measurements are acquired at $90^{\circ}$ resolution as the geoVISION tool rotates.

The geoVISION tool collar configuration is intended to run in $8 \frac{1}{2}$ inch $(22 \mathrm{~cm})$ and 9 inch $(25 \mathrm{~cm})$ diameter holes depending on the size of the measuring button sleeve. During Expedition 314, we used an $81 / 2$ inch diameter bit and an $81 / 4$ inch diameter button sleeve for the geoVISION tool. This resulted in a minimum standoff between the resistivity buttons and the formation, giving higher quality images.

\section{sonicVISION tool}

The sonicVISION sonic-while-drilling tool delivers real-time interval transit time data for compressional waves. The available measurement range is $\sim 40-230$ $\mu \mathrm{s} / \mathrm{ft}(1.3-7.6 \mathrm{~km} / \mathrm{s})$, depending on mud type, but in- tensive processing was sometimes required to obtain reliable sonic velocity measurements in the relatively slow formations drilled during Expedition 314. In real-time LWD operations, the sonic processing parameters are conventionally set at the surface before the tool is run in the hole. The real-time projection $\log$ and labeling with quality control log affect the real-time slowness and quality of the data. This results in the possible mislabeling of arrivals (especially for slow formations) and limited confidence levels, as only the end result of downhole processing is seen uphole in the real-time log. In general, during Expedition 314 the real-time sonic traveltimes were spurious and unreliable; however, full waveform data are recorded in memory. Advanced onboard postprocessing extended the range of measurement to near the mud velocity, a key feature for achieving the scientific objectives of this cruise, including logseismic ties. Additional quality control is performed using automatic stationary measurements made during a pipe connection. In this less noisy environment, the tool is able to take a station measurement that is sent uphole, when pumping resumes, for further quality control of the real-time log (Fig. F6).

The wideband frequency measurement and highpower transmitter have been improved from the older tool. Wideband frequency measurements reduce aliasing and eccentralization effects, improve formation signal strength, and allow measurement of shear and Stoneley waves. The transmitter outputs large amounts of wideband power to effectively couple more energy into various formation types, ultimately improving the signal-to-noise ratio and, therefore, measurement quality and hole size range. Extended battery life, large memory capacity, and fast dump speed significantly enhance the sonicVISION tool's reliability and functionality. Standard memory life is $140 \mathrm{~h}$ at $10 \mathrm{~s}$ acquisition, and this is easily doubled. In addition, standardized "planning" software allows the engineer to easily optimize the tool configuration for the well being drilled.

In shallow unconsolidated formations where the compressional velocity approaches or is below the fluid (mud) velocity, it is difficult to directly measure the formation slowness with the refracted wave because the energy of the refracted wave is too weak. When the compressional slowness is larger than the mud slowness, a significantly low frequency (several kilohertz) source is needed to measure the formation slowness (Wu et al., 1995). However, if the compressional slowness is close to the mud slowness but still smaller than the mud slowness, the formation slowness can be extracted with processing a leaky compressional ("leaky-P") mode excited by a wideband 
source. Tichelaar and Luik (1995) and Valero et al. (1999) discussed the compressional slowness processing in such conditions for wireline sonic data. They processed a leaky-P mode by applying a lower frequency band-pass filter to attenuate fluid modes and enhance formation arrivals. The leaky-P mode consists of multiple reflected and constructively interfering compressional waves traveling in the borehole fluid (Paillet and Cheng, 1991). The leaky-P mode is dispersive, such that at lower frequencies the slowness asymptotically approaches the formation compressional slowness and at higher frequency to the mud slowness. Because of this dispersive effect, the slowness estimated by the nondispersive semblance processing (Kimball and Marzetta, 1984) is greater than the true $P$-wave slowness. Therefore, correction for dispersion is needed in order to obtain the true formation compressional slowness.

Standard LWD-sonic measurements are operated with a frequency band of $\sim 11 \mathrm{kHz}$ (Aron et al., 1997). However, in the case of a very slow formation, it is difficult to obtain the compressional slowness using a standard source because the energy of the fluid arrivals dominates those of the leaky-P mode. It is necessary to expand the source spectrum of the monopole transmitter to lower frequencies to excite the leaky-P mode. Therefore, wide frequency band data acquisition is required to excite a leaky-P mode. Both the semblance processing and the dispersive analysis with Prony method (Ekstrom, 1995) clearly showed the existence of dispersive leaky modes.

The modeling of leaky-P dispersion has to take into account a realistic tool structure for a wide range of borehole and formation parameters. Based on these modeling results, a correction table for the dispersion biases is established and dispersion correction applied to obtain formation compressional slowness. This procedure was applied to the ODP logs from Leg 196 Hole 1173B and Leg 130 Hole 808I and compared with the core slownesses measured on the previous leg (Mikada et al., 2002). The result of the dispersion-corrected LWD sonic processing showed good agreements with these core velocity measurements (Goldberg et al., 2005). A similar procedure was applied by the Schlumberger Data Consulting Service (DCS) specialist for onboard processing of sonic data.

\section{seismicVISION tool}

The seismicVISION LWD system delivers time- and depth-velocity information to provide interval velocity. The seismicVISION tool, which contains a processor and memory, receives seismic energy from a conventional air gun suspended from a crane on the drillship. After acquisition, the seismic signals are stored and processed downhole, and check shot data and quality indicators are transmitted uphole in real time by connection with the MWD pulse system. Waveforms are recorded in the tool memory for further processing after a bit trip. Refer to "Log-seismic correlation" for more information (Fig. F7).

\section{Onboard data flow and quality check}

For each operation, two types of data are collected: (1) real-time data that include all MWD-APWD data and selected LWD data and (2) LWD data that have been recorded downhole and stored in the tool's memory. Data are originally recorded downhole at a preset frequency. The depth version is obtained after merging the time (downhole) with the time-depth relationship recorded on the surface by the IDEAL system. For the MWD-APWD, adnVISION, and geoVISION tools, both time and depth versions of the data exist. The raw sonicVISION data were only distributed versus depth and were processed onboard by the DCS specialist. In the same way, the seismicVISION data (check shots) were only available versus depth and distributed to the Shipboard Science Party for immediate processing and analysis. The time version of the data was made available in log ASCII standard (LAS) format. The depth version of the data was made available in digital logging interchange standard (DLIS) format for the MWDAPWD, adnVISION, geoVISION, and sonicVISION tools and in Society of Exploration Geophysicists standard (data format " $\mathrm{Y}$ ") for the seismicVISION tool.

After determining the position of the mudline by identifying a break in the gamma ray log (and resistivity logs, when available), the data were depth shifted to the seafloor (LWD depth below seafloor [LSF]). The depth-shifted version of the MWDAPWD, adnVISION, geoVISION, and processed data were distributed in the native format (DLIS), and the main scalar logs were extracted and converted into LAS files. All files (time based, depth based, original, and depth shifted) and associated documentation (quality check and operation reports) were distributed to the Shipboard Science Party through the onboard intranet data servers. Analyses, integration results, and reports produced by the Shipboard Scientific Party were then archived on the server for further distribution. Normal data flow is illustrated in Figure F8.

The Logging Staff Scientist performed initial conversion and output of the raw data received from the Wellsite Geologist, who received the data from the Schlumberger LWD engineer. Logging Staff Scientist 
duties include documentation of the MWD-APWDLWD operations, data quality assessment (highlighting any abnormalities), depth shifting (i.e., converting depth below rig floor to depth below seafloor), and systematic distribution and documentation of data. Operations and quality assessment are described in two main plots (data versus time and data versus depth) related by a third time-depth relationship plot (Fig. F9). In the first plot (Plot 1), timedepth relationship (Panel 1.1), surface drilling and downhole parameters (Panel 1.2), and selected geophysical logs (Panel 1.3) are plotted versus time to identify the sequence of drilling events and further assess their possible impact on data quality. In the second plot (Plot 2), the time-time relationship (Panel 2.1) and operational surface and downhole data (Panel 2.2) are reported versus depth for largescale assessment of drilling conditions on data quality. These first panels are completed by detailed analysis of the ultrasonic caliper, density, correction on bulk density, and comparison of the shallow and deep button resistivity scalar logs to further assess hole condition (cave, washout, or bridge) and possible impact on density/porosity data, as well as invasion (Panel 2.3). Elapsed time of the main geophysical measurements after bit is also indicated in this third panel. In a fourth panel (Panel 2.4), changes in parameters of the sonic processing and a quality indicator of the resulting processed sonic log are documented. Finally, results of detailed quality assessment of borehole images (mostly shallow, medium, and deep button resistivity and gamma ray images) are documented in the last panel (Panel 2.5). The third time-depth relationship plot (Plot 3), made at the same scale as the two main time and depth plots, allows easy navigation between those two plots.

Quality of MWD-APWD-LWD data is mostly assessed by cross-correlating available logs. Available logs are of two types, as follows:

1. Drilling control logs including surface drilling surface parameters (e.g., ROP, surface weight on bit [SWOB], hook load [HKLD], standpipe pressure [SPPA]), and downhole drilling parameters (e.g., collar [bit] rotation [CRPM], hole deviation [HDEVI], radial shock rate [SKR_R], tangential shock rate [SKR_T], shock peak [SHKPK], and stick-slip indicator [SLIP]) and

2. Geophysical control logs such as calipers (ADIA, ECAL_RAB), gamma ray (GR), annular pressure (APWD), and temperature (ATMP_MWD).

Geophysical logging data may be degraded where borehole diameter greatly increases or is washed out. Deep investigation measurements such as resistivity and sonic velocity are least sensitive to borehole conditions. Nuclear measurements (density and neutron porosity) are more sensitive because of their shallow depth of investigation and the effect of drilling fluid volume on neutron and gamma ray attenuation. Corrections were applied to the original data to reduce these effects. The effects of very large washouts, however, cannot be corrected.

Azimuthal measurements and associated images are of low quality when the tool is not rotating (slip) or when its rotation exceeds $250 \mathrm{rpm}$. In zones of high stick-slip, even if tool rotation (CRPM) is set to a typical value of $100 \mathrm{rpm}$, CRPM can greatly vary locally (and exceed $250 \mathrm{rpm}$ ), resulting in images of lower quality. As all measurements even by the same tool are not sampled at the same time (sampling rate of adnVISION and geoVISION = $5 \mathrm{~s}$ ), improper heave compensation and irregular movement of the BHA (vibration shocks or bending) can result in local depth shift between measurements by several tens of centimeters.

\section{Log characterization and lithologic interpretation}

LWD measurements provide in situ petrophysical information on rocks and pore fluids while the hole is being drilled. These measurements are sensitive to changes in composition (changing curve magnitudes), textures, and structures (log shape, peak amplitude, and frequency, as well as information from image logs). Changes in the log response (values and/or frequency of the signal) are commonly associated with geological unit boundaries.

This section addresses the characterization of the LWD measurements and imaging tool response, focusing on zoning the well logs into logging units. Once representative petrophysical properties for the logging units were defined, they were incorporated in the log-based lithologic units. This process can be achieved by qualitative and quantitative methods.

\section{Log characterization and identification of logging units \\ Qualitative analysis}

The geometry of logging unit boundaries and bedding information was defined on the basis of borehole images and characterized from scalar LWD logs. Rock textures and structures were analyzed on borehole images, and vertical trends were analyzed on all the available logs. Composition and textural information was derived mainly from nuclear (spectral gamma, density, and PEF) and sonic logs. Data quality assessment was made by shipboard scientists through the examination of the potential effect of borehole diameter and conditions and drilling pa- 
rameters on the logs prior to interpretation of the log response.

The first qualitative approach to unit definition was identification of the boundaries separating sections of different $\log$ responses and concomitant rock properties. For this type of analysis, natural- and induced-radioactivity logs, sonic logs, resistivity, and borehole images were the main input.

Integrated interpretation of all the available logs focused on the following items:

- Definition and characterization of logging units, subunits, and unit boundaries;

- Identification of compositional features within each unit; and

- Interpretation in terms of geological features (unit boundaries, transitions, sequences, and likely lithologic composition).

\section{Quantitative analysis}

As a consistency check of the qualitative interpretation and for quantitative log characterization, we applied statistical grouping. This involved investigating the percentile ranges and distribution of absolute values within the visually defined logging units.

\section{Log-based geological/lithologic interpretation}

During Expedition 314, lithologies were interpreted based only on LWD logs without cores, unlike previous ODP/IODP expeditions. After log characterization and classification, logs were lithologically and geologically interpreted using a combination of $\log$ characteristics and borehole images for each site.

Compositionally influenced logs such as gamma ray and PEF logs were used to determine lithology from unit scale to bed scale. In particular, the identification of sand-rich intervals, clay-rich intervals, or alternating beds of sand and clay was a primary element of the interpretation. Sonic and all other logs were also used for lithology characterization. Borehole images provided useful information on mesoscopic features such as bedding, sedimentary structures, bed boundaries, unconformities, and faults. Additionally, lithologic information from ODP Legs 131, 190, and 196, all in the Nankai Trough off Shikoku Island, was an important aid in interpreting lithology.

These interpretations will be confirmed by correlation with core data from subsequent NanTroSEIZE project expeditions. A possible correlation to seismic units was also proposed for each site.

\section{Physical properties}

The principal objectives for physical property analysis of the logging data concern the mechanical state and physical properties of sediments in the accretionary prism, Shikoku Basin, and the trench fill, as well as in the tectonic features. It also concerns the assessment of hydrogeologic conditions inferred on the basis of physical property data.

The standard downhole logs provide information on a wide range of in situ physical properties. This includes $P$-wave velocity, electrical resistivity, gamma ray intensity, bulk density, and porosity.

In each site chapter, the physical properties section presents the logs mentioned above as a function of depth and describes their features and their variation in conjunction with lithology, structural geology, and $\log$-seismic integration. The bulk density log is plotted mainly using adnVISION image-derived bulk density (Schlumberger mnemonic IDRO) data. When IDRO data were not available, bulk density (RHOB) was used instead. The porosity log is plotted using thermal neutron porosity (TNPH) and is derived from bulk density for comparison. Ring; bit; and shallow, medium, and deep button resistivity logs in different measurement configurations are compared against each other to examine measurement conditions and applied corrections. $P$-wave velocity is calculated and plotted as the inverse of compressional wave slowness from the Schlumberger sonicVISION tool (DTCO).

\section{Estimation of porosity from the density log}

A density-derived porosity log is calculated from the bulk density log using the assumption of a constant grain density $\left(\rho_{\mathrm{g}}\right)$ of $2.65 \mathrm{~g} / \mathrm{cm}^{3}$ and a constant water density $\left(\rho_{\mathrm{w}}\right)$ of $1.024 \mathrm{~g} / \mathrm{cm}^{3}$ (Blum, 1997). In the absence of grain density measured on cores from the same site, we based the value of the constant density on previous measurements in the Nankai accretionary prism area off Muroto during Leg 190 (Moore, Taira, Klaus, et al., 2001). The equation used to derive the porosity $(\varphi)$ from the bulk density $\log \left(\rho_{b}\right)$ is

$$
\varphi=\frac{\rho_{\mathrm{b}}-\rho_{\mathrm{g}}}{\rho_{\mathrm{w}}-\rho_{\mathrm{g}}} .
$$

\section{Estimation of porosity from the resistivity log}

Archie's law (Archie, 1947) is usually used to derive a porosity log from the resistivity log as 


$$
F=\frac{a}{\varphi^{m}}
$$

where $F$ is the formation factor, $a$ is a constant, and $m$ is the so-called cementation factor. The variable $m$ depends on rock type and is more closely related to texture than to cementation. Several values of $a$ and $m$ can be found in the literature. The actual values of the $a$ and $m$ parameters will be explained in each site chapter. One limitation of this simple approach is that it does not take lithology or pore fluid variations into account. It should also be noted that the resulting estimate is very sensitive to the choice of Archie's law constants.

The formation factor is calculated as

$$
F=\frac{R}{R_{\mathrm{f}}}
$$

where $R$ is the LWD-measured resistivity and $R_{\mathrm{f}}$ is the fluid resistivity. We assumed that the pore fluid is similar to seawater. The formula used to calculate the resistivity of seawater $\left(R_{\mathrm{f}}\right)$ as a function of temperature $T\left({ }^{\circ} \mathrm{C}\right)$ is as follows (Shipley et al., 1995):

$$
R_{\mathrm{f}}=\frac{1}{2.8+0.1 T} \text {. }
$$

The temperature profile was calculated at each site considering a conductive heat transfer, the measured surface heat flux, and an estimation of the thermal conductivity based on previous coring in the Nankai accretionary prism. The value used for this calculation is defined in each site chapter.

The bit resistivity measurement was used for this estimation because this is the measurement with the largest depth of investigation (12 inches) and because the position at the bit of the tool string minimizes the effect of formation modification induced by drilling.

\section{Structural geology and geomechanics}

Structural analysis was performed primarily on geoVISION resistivity images using GMI Imager (Geomechanics International Inc.), GeoLog/Geomage (Paradigm Geotechnology B.V.), and GeoFrame (Schlumberger) software. These software packages present resistivity image data of the borehole wall as a planar "unwrapped" $360^{\circ}$ image with depth. The software also allows visualization of the data in a three-dimensional (3-D) borehole view.

The geoVISION downhole tool provides resistivity images of shallow, medium, and deep depths of investigation, imaging the formation at $\sim 34, \sim 43$, and $\sim 55 \mathrm{~cm}$ diameters of investigation, respectively. Re- sistivity image data were imported into the software packages and displayed as both statically and dynamically normalized images. Static normalization displays the image with a color range covering all resistivity values for the entire logged interval. Static normalization is preferred for comparing relative changes in resistivity throughout the borehole and is therefore ideal for correlating lithologic or facies changes and comparing the resistivity of particular fault zones. Dynamic normalization scales the color range for resistivity values over a specified interval. Dynamic normalization is commonly used for detailed identification of fractures and sedimentary structures and often allows more subtle changes in the image to be identified. Further filtering of the data was also possible with the software. Image data were compared with borehole caliper data to investigate the changing average diameter of the borehole.

Methods of interpreting structure and bedding differ considerably between cores, wireline logs, and LWD data sets. Horizontal and vertical resolution of resistivity images is considerably lower than comparable data from cores and wireline image logs (e.g., Fullbore Formation MicroImager [FMI]). Vertical resolution for LWD resistivity images is $\sim 5-7.5 \mathrm{~cm}$ if ROP is maintained at $\sim 20-30 \mathrm{~m} / \mathrm{h}$. Horizontal resolution across the image is a function of several factors. Some of these factors cannot be precisely constrained; therefore, this resolution has some uncertainty. These factors include the diameter of investigation (i.e., shallow, medium, or deep), which is also influenced by borehole elongation; the difference between formation resistivity and that of the borehole fluid; and the number of measurements made around the hole (56 for the geoVISION tool). The ratio between $R_{\mathrm{t}}$ (true resistivity of formation) and $R_{\mathrm{xo}}$ (resistivity of zone invaded by drilling fluid) also influences the diameter of investigation, but we assumed the ratio to be 1.0, owing to minimal invasion because resistivity was measured soon after drilling. For the geoVISION tool and the likely range of formation resistivities encountered during this expedition, the approximate horizontal resolutions range from $\sim 2$ to $3 \mathrm{~cm}$. The ability to image a feature (feature detection) is also a function of the resistivity contrast and resolution. It may therefore be possible to resolve features smaller than the expected vertical or horizontal resolution if they contrast strongly with the background resistivity. The vertical resolution also controls whether the thickness of a layer can be determined. These resolutions should be compared with cores (millimeters) and FMI wireline resistivity images $(\sim 0.5 \mathrm{~cm})$; therefore, smaller features are not resolvable within the LWD images. For example, individual microfaults ("small faults" $<1$ 
$\mathrm{mm}$ width) and shear bands (1-2 $\mathrm{mm}$ to $1 \mathrm{~cm}$ in width) identified in cores (e.g., Site 808) should not be resolvable in LWD resistivity image data. This should be considered when directly comparing reports from previous and future data sets.

In the unwrapped geoVISION resistivity images, sinusoidal lines are planar surfaces inclined to the borehole axis. Curved lines differing from sinusoids are nonplanar surfaces. To pick planar features (bedding planes, beds, fractures, faults, etc.), sinusoids were interactively fitted to determine dip and azimuth. Features were further classified according to type of fracture, width/aperture, shape, and relative resistivity (conductive versus resistive). Further analysis (fracture frequency, azimuth distribution, etc.) was performed in GMI Imager, GeoLog/Geomage, GeoFrame, and other software packages. We also compared resistivity images directly with other logging data for interpretation of bedding planes and for correlation of deformation style, lithology, and physical properties.

We identified fractures within geoVISION resistivity images by their contrasting resistivity or conductivity, from contrasting dip relative to surrounding bedding trends, or by truncation of other features. Resistivity of fractures is defined relative to the full range of resistivity values within the hole and is nonquantitative. If relative resistivity is unclear, the fracture resistivity is undefined. In some cases, conductive fractures may be easier to identify relative to the background resistivity, biasing the results slightly. Differentiation between fractures and bedding planes is complex and less accurate where bedding is inclined. Care was taken to avoid misinterpreting borehole artifacts as natural geological features. We compared initial structural interpretations from the logging data with seismic reflection data. Comparisons with cores will be possible following later expeditions. Our interpretations are based on the above criteria, but we acknowledge that some fractures and bedding planes may have been misinterpreted.

\section{Borehole wall failure analysis}

Breakouts and/or tensile fractures, two types of drilling-induced borehole wall failure, form when the state of local stress field at the borehole wall exceeds rock/sediment strength. Breakouts form parallel to the minimum principal horizontal stress $\left(S_{\text {hmin }}\right)$ and perpendicular to the maximum horizontal principal stress $\left(S_{\text {Hmax }}\right)$, resulting in elongation of the borehole. Breakouts are recorded in resistivity images as two parallel conductive vertical features $180^{\circ}$ apart. Drill- ing-induced tensile fractures may form in conjunction with breakouts or independently. The tensile fractures form perpendicular to $S_{\mathrm{hmin}}, 90^{\circ}$ from the azimuth of the breakouts.

We recorded the orientation, downhole extent, and width of breakouts with the image analysis software considering all three borehole images (shallow, medium, and deep). Caliper data allow visualization of the changing average borehole diameter with depth, but azimuthal caliper data were not referenced to geographic coordinates and so cannot be used to assess borehole elongation. We compared breakout distribution and width with lithology (from image resistivity and defined lithologic units derived from all logging data) and drilling parameters. Further breakout analysis was conducted with GMI and other software.

\section{Constraining stress from drilling-induced tensile fractures}

Drilling-induced tensile fractures occur when the hoop stress at the borehole wall exceeds rock tensile strength. Where the tensile strength of sediments is negligible, the occurrence of drilling-induced tensile fractures is an indicator of tensile hoop stress at the borehole wall. We attempted to estimate in situ stress magnitudes by constraining possible stress ranges that allow failure in the borehole, specifically the formation of drilling-induced tensile fractures. Most of the following discussion is drawn from Zoback et al. (2003).

Because the stress in sediment is limited by the strength of frictional sliding on faults, it is possible to constrain the range of possible stress states at any depth and pore pressure. If the ratio between the two extreme effective principal stresses goes beyond certain values defined by the coefficient of friction, sliding occurs along critically oriented faults (Byerlee, 1978), which in turn releases the excess stresses. More explicitly, the limiting condition for failure can be expressed by Coulomb friction law:

$$
\frac{S_{1}-P_{\mathrm{P}}}{S_{3}-P_{\mathrm{P}}}=\left(\sqrt{\mu^{2}+1}+1\right)^{2},
$$

where $\mu^{2}$ is the coefficient of friction, $P_{\mathrm{p}}$ is pore pressure, and $S_{1}$ and $S_{3}$ are the maximum and minimum in situ principal stresses, respectively. This equation can be plotted as three lines (1, 2, and 3 in Fig. F10) representing the conditions of failure and defining the "stress polygon" in the $S_{\mathrm{H} \max }$ versus $S_{\mathrm{hmin}}$ or horizontal stress domain. This stress polygon encompasses the possible states of stress at a given depth. 
Above and to the left of these three lines the sediment/rock would be at failure in its natural state. The interiors of the stress polygons define allowable values for horizontal principal stresses for conditions favoring but not at the threshold of normal, strikeslip, and thrust faulting. The size of the stress polygon depends on the coefficient of friction, depth, and pore pressure.

IODP holes provide information on the vertical stress from the overburden, borehole pressure by direct downhole measurements with MWD tools, failure through the presence of breakouts and drillinginduced tensile fractures, and potentially, style of deformation from offset features. These observations are used with the theoretical framework above to provide estimates of the stresses in the borehole and whether the stress ratios favor normal, thrust, or strike-slip faulting.

Estimation of stress magnitude is possible when information on rock strength is available. Since no core was recovered from this LWD expedition, indirect estimations of rock strength parameters (unconfined compressive strength and internal friction coefficient) were made using a set of empirical equations that relate rock strength to other physical properties measured from geophysical logging (Chang et al., 2006). The two strength parameters are sufficient to construct any well-known rock strength criteria (Colmenares and Zoback, 2002). We used a strength criterion that is suitable for describing the general strength characteristics of the sedimentary rock. Ranges of possible in situ stress magnitudes were constrained by comparing rock strength with the state of the local stress field at the borehole wall where we observed borehole breakouts.

\section{Log-seismic correlation}

During Expedition 314 we used several of the LWD data sets to establish accurate ties to the $2006 \mathrm{Ku}-$ mano 3-D seismic reflection data set (Moore et al., 2007). Data from seismicVISION and sonicVISION tools helped to establish a traveltime to depth transform at the boreholes.

\section{seismicVISION tool}

The seismicVISION tool produces data which can be interpreted as a check shot survey, a low-resolution velocity depth function, and a vertical seismic profile. The seismicVISION tool records seismograms using a hydrophone and a three-component geophone in the tool and a surface source and hydro- phone (www.slb.com/content/services/drilling/ imaging/seismicvision.asp). The source was three 250 in $^{3}$ air guns (Fig. F11) that were suspended from crane $1 \sim 55 \mathrm{~m}$ horizontally from the rotary table (Fig. F12) and fired $6 \mathrm{~m}$ below sea level at 17002000 psi. Time correlations of the shots are ensured using high-precision clocks at both the surface hydrophone and downhole hydrophone. The surface hydrophone was suspended $3 \mathrm{~m}$ below the air guns (total $9 \mathrm{~m}$ below mean sea level) and the zero times of the waveforms were corrected to mean sea level.

Data cannot be usefully obtained when the pipe is rotating or moving vertically or when the drilling fluid pumps are running. Therefore, seismicVISION data are typically acquired at each addition or removal of a stand of drill pipe ( $38 \mathrm{~m}$ long) to or from the drill string. The Chikyu uses four joints $(9.5 \mathrm{~m}$ long drill pipe) as one stand. The drill string is stationary and the pumps are off during these times. This means that while drilling the hole, data were acquired every $38 \mathrm{~m}$. After drilling was complete and while the pipe was being recovered, data were also acquired every $38 \mathrm{~m}$ but at points shifted by two joints $(19 \mathrm{~m})$ relative to the acquisition during drilling. Thus, combining the data acquired during drilling and the data acquired during pipe recovery yielded data nominally acquired at $19 \mathrm{~m}$ intervals in the borehole (Fig. F13).

At each data acquisition level, a number of shots were fired by the surface source. When rig circulation/rotation stopped, the seismicVISION acquisition system was activated. The source was fired at 15 $s$ intervals. The time for the insertion or removal of a pipe stand was at least $3 \mathrm{~min}$. Thus, the standard procedure was to fire 10-15 shots. The tool records the pressure (hydrophone) and acceleration (threecomponent geophones) seismograms obtained for each shot and calculates and records a vertical stack of the shots. In practice, because our holes were near vertical and the tool was centered and unclamped, the geophone data yielded little useful information. The tool also automatically picks and records the $P$ wave arrival time in the stacked seismogram. The stacked seismogram and the picked $P$-wave arrival times from the instrument are transmitted to the ship by the MWD system and thus could be used soon after acquisition at each level or as the primary data in case the tool fails to record data or is lost.

A check shot survey consists of the one-way first arrival traveltimes of a seismic pulse from the surface to a downhole hydrophone placed at a series of known depths in the hole. This corresponds to the picked $P$-wave arrival at each depth from the seis- 
micVISION tool. This was the most reliable data set for matching seismic reflectors acquired in the time domain with logging and core data acquired in the depth domain. The check shot data are a key input to the process of making a synthetic seismogram.

We processed the seismicVISION data using ProMAX software (Landmark-Halliburton). After manual editing of bad or noisy traces and frequency filtering, the shots from each level were stacked and first breaks were picked.

A velocity depth function was obtained by taking the differences between adjacent vertical traveltime data (the check shot data) and dividing by the depth difference. Velocity depth curves obtained in this manner are typically quite noisy. A smooth version of this curve was obtained using the methodology of Lizarralde and Swift (1999). This curve may be usefully compared with the velocity versus depth data from the sonicVISION tool and with the velocity versus depth at the hole location used in the processing of the 3-D seismic volume.

A vertical seismic profile can be obtained by assembling a gather of the stacked seismograms from a hole sorted by receiver depth. This gather was filtered to remove noise and the upgoing and downgoing waves were separated using FK filtering. The upgoing arrivals were then moved out (flattened in time) and stacked to produce an equivalent of the zero-offset reflection seismogram, called a corridor stack, at the hole location. When the seismicVISION data are of very high quality, this stack should compare favorably with the hole location trace in the 3-D multichannel seismic (MCS) reflection volume. Differences in arrival times between these seismograms may indicate problems with the velocity field used to depth migrate/convert the MCS data.

\section{Depth conversion of seismic reflection data}

We used the time-depth transforms obtained from the seismicVISION data to convert the original prestack time-migrated 3-D seismic reflection data to the depth domain for correlation with the LWD data. For each site we made depth conversions of an inline seismic section that passes through the drill site (100 traces on each side of the site using ProMAX seismic processing software).

\section{Synthetic seismograms}

We constructed synthetic seismograms using the best available density curve and the detailed slowness (inverse of velocity) log from the sonicVISION tool. Where the sonicVISION tool was not working properly, because of slow formations or other complications, we generated a velocity curve from the density log using the Gardner equation (Gardner et al., 1974).

Conversion from time to depth was required during synthetic seismogram construction to allow correlation of the depth-based LWD logs to the traveltimebased seismic data. This conversion was done using the check shot data generated by the seismicVISION tool to generate a traveltime to depth relationship.

The sonicVISION tool provides good information for interval velocity changes at short wavelengths. The seismicVISION tool, after processing and filtering, provides a reliable smooth interval velocity curve at long wavelengths. Following general industrial practices, we took the smoothed interval velocity curve from the check shot as correct and calibrated the sonic log so that these two curves matched in their time to depth relationships. This calibration is achieved by creating a drift curve that shifts the sonic log within defined intervals (based on inflection points in the drift curve) while preserving the shorter wavelength relative velocity changes provided by the sonic data.

To create a synthetic seismogram, a source wavelet was convolved with a reflectivity series using GeoFrame software (Schlumberger). The reflectivity is expressed in the following form:

$$
R=\left(v_{2} \rho_{2}-v_{1} \rho_{1}\right) /\left(v_{1} \rho_{1}+v_{2} \rho_{2}\right),
$$

where $v_{1}, v_{2}$ and $\rho_{1}, \rho_{2}$ are the acoustic velocity and density in the upper layer and lower layer, respectively. For Expedition 314, we estimated the source wavelet from the best waveform and amplitude match provided by wavelets extracted within 20 traces of each site in the orientation (inline or crossline) providing the flattest seafloor. We used a deterministic extraction method based either on the energy or power spectrum and in each case let the software define the best wavelet length and lag. To obtain a wavelet we defined a start and end time within the trace, always starting shallower than the seafloor and ending deeper than the wavelet length. The software computes a zero lag autocorrelation of the reflection coefficients from each trace within the region defined and then computes a signal-to-noise ratio for each trace. An optimal inline/cross-line pair is returned along with an optimal time lag that results in the highest signal-to-noise ratio and that passes a 90\% confidence level based on normalized mean square error (NMSE). We determined the optimal wavelet length by computing the best length at 
each site using the portion of energy predicted (PEP) method. In this method, a wavelet is generated based on the deterministic method (power spectrum comparison or energy comparison) for each wavelength to be tested and then convolved with the reflection coefficient to generate a synthetic seismogram. The resulting synthetic seismogram is then compared to the input trace based on its PEP and the best match wavelet length is returned. The optimum wavelet lengths in all cases proved to be $256 \mathrm{~ms}$, the lags varied some but were usually close to zero, and the extracted wavelets were all zero phase. For each site, we varied the start and end time for the wavelet extraction window and tried both energy and power spectrum comparisons until we attained a source wavelet that had high signal-to-noise ratio, passed the NMSE test, and visually provided the best fit to the frequency spectrum of the input data.

Using the check shot curve, calibrated sonic log, and best available density log we were able to create a reflection coefficient series. This series was then convolved with the extracted source wavelet to generate a synthetic seismogram at a $4 \mathrm{~ms}$ sampling interval. Displaying the synthetic seismogram beside the seismic data from the area of the borehole provides information about specific boundaries of interest and a quality check on velocity and density logs.

\section{References}

Anadrill-Schlumberger, 1993. Logging While Drilling: Houston (Schlumberger), SMP-9160.

Archie, G.E., 1947. Electrical resistivity-an aid in core analysis interpretation. AAPG Bull., 31:350-366.

Aron, J., Chang, S.K., Codazzi, D., Dworak, R., Hsu, K., Lau, T., Minerbo, G., and Yogeswaren, E., 1997. Real-time sonic logging while drilling in hard and soft rocks. Trans. SPWLA Annu. Logging Symp., 38:1HH-14HH.

Blum, P., 1997. Physical properties handbook: a guide to the shipboard measurement of physical properties of deep-sea cores. ODP Tech. Note, 26. doi:10.2973/ odp.tn.26.1997

Byerlee, J., 1978. Friction of rocks. Pure Appl. Geophys., 116(4-5):615-626. doi:10.1007/BF00876528

Chang, C., Zoback, M.D., and Khaksar, A., 2006. Empirical relations between rock strength and physical properties in sedimentary rocks. J. Pet. Sci. Eng., 51(3-4):223-237. doi:10.1016/j.petrol.2006.01.003

Colmenares, L.B., and Zoback, M.D., 2002. A statistical evaluation of intact rock failure criteria constrained by polyaxial test data for five different rocks. Int. J. Rock Mech. Min. Sci., 39(6):695-729. doi:10.1016/S13651609(02)00048-5

Ekstrom, M.P., 1995. Dispersion estimation from borehole acoustic arrays using a modified matrix pencil algorithm. Signals, Syst. Comput., 1:449-453. doi:10.1109/ ACSSC.1995.540589
Flemings, P.B., Behrmann, J.H., John, C.M., and the Expedition 308 Scientists, 2006. Proc. IODP, 308: College Station TX (Integrated Ocean Drilling Program Management International, Inc.). doi:10.2204/ iodp.proc.308.2006

Gardner, G.H.F., Gardner, L.W., and Gregory, A.R., 1974. Formation velocity and density-the diagnostic basics for stratigraphic traps. Geophysics, 39(6):770-780. doi:10.1190/1.1440465

Goldberg, D., Cheng, A., Gulick, S., Blanch, J., and Byun, J., 2005. Velocity analysis of LWD sonic data in turbidites and hemipelagic sediments offshore Japan, ODP Sites 1173 and 808. Proc. ODP, Sci. Results, 190/196: College Station TX (Ocean Drilling Program), 1-15. doi:10.2973/odp.proc.sr.190196.352.2005

Kimball, C.V., and Marzetta, T.L., 1984. Semblance processing of borehole acoustic array data. Geophysics, 49(3):274-281. doi:10.1190/1.1441659

Lizarralde, D., and Swift, S., 1999. Smooth inversion of VSP traveltime data. Geophysics, 64(3):659-661. doi:10.1190/1.1444574

Mikada, H., Becker, K., Moore, J.C., Klaus, A., et al., 2002. Proc. ODP, Init. Repts., 196: College Station, TX (Ocean Drilling Program). doi:10.2973/odp.proc.ir.196.2002

Moore, G.F., Bangs, N.L., Taira, A., Kuramoto, S., Pangborn, E., and Tobin, H.J., 2007. Three-dimensional splay fault geometry and implications for tsunami generation. Science, 318(5853):1128-1131. doi:10.1126/science. 1147195

Moore, G.F., Taira, A., Klaus, A., et al., 2001. Proc. ODP, Init. Repts., 190: College Station, TX (Ocean Drilling Program). doi:10.2973/odp.proc.ir.190.2001

Moore, J.C., and Klaus, A. (Eds.), 2000. Proc. ODP, Sci. Results, 171A: College Station, TX (Ocean Drilling Program). doi:10.2973/odp.proc.ir.171A.1998

O'Brien, P.E., Cooper, A.K., Richter, C., et al., 2001. Proc. ODP, Init. Repts., 188: College Station, TX (Ocean Drilling Program). doi:10.2973/odp.proc.ir.188.2001

Paillet, F.L., and Cheng, C.H., 1991. Acoustic Waves in Boreholes: Boca Raton, FL (CRC Press).

Radtke, R.J., Adolph, R.A., Climent, H., Ortenzi, L., and Wijeyesekera, N., 2003. Improved formation evaluation through image-derived density. Petrophysics, 44(2):137138.

Riedel, M., Collett, T.S., Malone, M.J., and the Expedition 311 Scientists, 2006. Proc. IODP, 311: Washington, DC (Integrated Ocean Drilling Program Management International, Inc.). doi:10.2204/iodp.proc.311.2006

Schlumberger, 1989. Log Interpretation Principles/Applications: Houston (Schlumberger Educ. Services), SMP7017.

Schlumberger, 1994. Log Interpretation Charts: Sugarland, TX (Schlumberger Wireline and Testing), SMP-7006.

Shipley, T.H., Ogawa, Y., Blum, P., et al., 1995. Proc. ODP, Init. Repts., 156: College Station, TX (Ocean Drilling Program). doi:10.2973/odp.proc.ir.156.1995

Tréhu, A.M., Bohrmann, G., Rack, F.R., Torres, M.E., et al., 2003. Proc. ODP, Init. Repts., 204: College Station, TX 
(Ocean Drilling Program). doi:10.2973/ odp.proc.ir.204.2003

Tichelaar, B.W., and van Luik, K.W., 1995. Sonic logging of compressional wave velocities in a very slow formation. Geophysics, 60(6):1627-1633. doi:10.1190/1.1443895

Valero, H.P., Brie, A., Pistre, V., and Higgins, T., 1999. Evaluation of slowness in slow formations [paper presented at the 5th Well Logging Symposium of the Society of Professional Well Log Analysts, Japan Chapter, Chiba, Japan, 29-30 September 1999]. $\phi$
Wu, P.T., Darling, H.L., and Scheibner, D., 1995. Low-frequency $P$-wave logging for improved compressional velocity in slow formation gas zones. SEG Annu. Meet. Expanded Tech. Program Abstr. Biogr., 65:9-12.

Publication: 5 March 2009

MS 314315316-112 
Figure F1. Drill string configuration used for (A) measurement-while-drilling (MWD)-APWD-LWD operations and (B) MWD-annular pressure while drilling (APWD). OD = outer diameter, $\mathrm{GR}=$ gamma ray, $\mathrm{NM}=$ nonmagnetic, $\mathrm{PNMDC}=$ pony nonmagnetic drill collar, FG NB = fine gauge near bit.

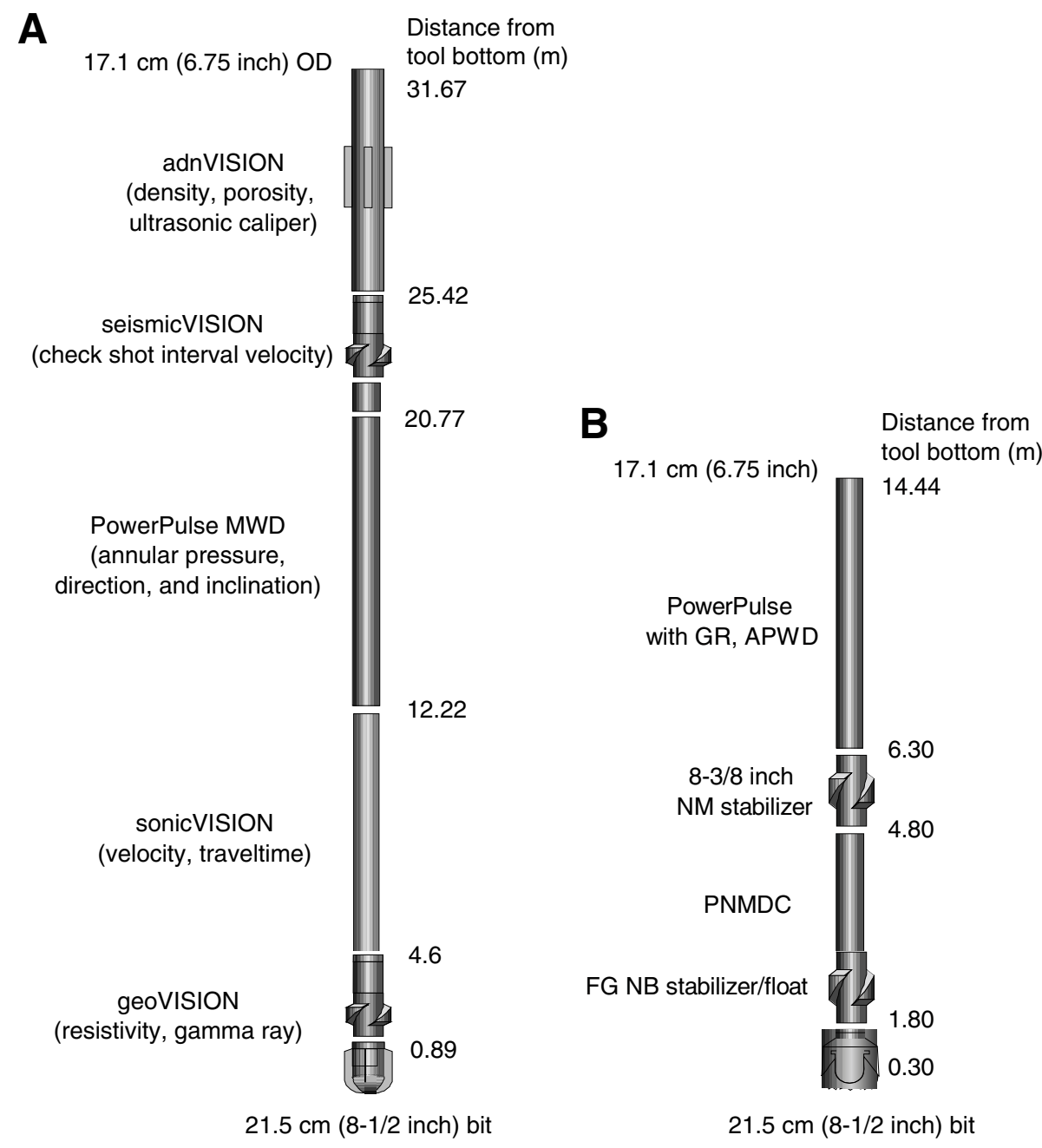


Figure F2. Schematic figure of rig instrumentation. MWD = measurement while drilling.

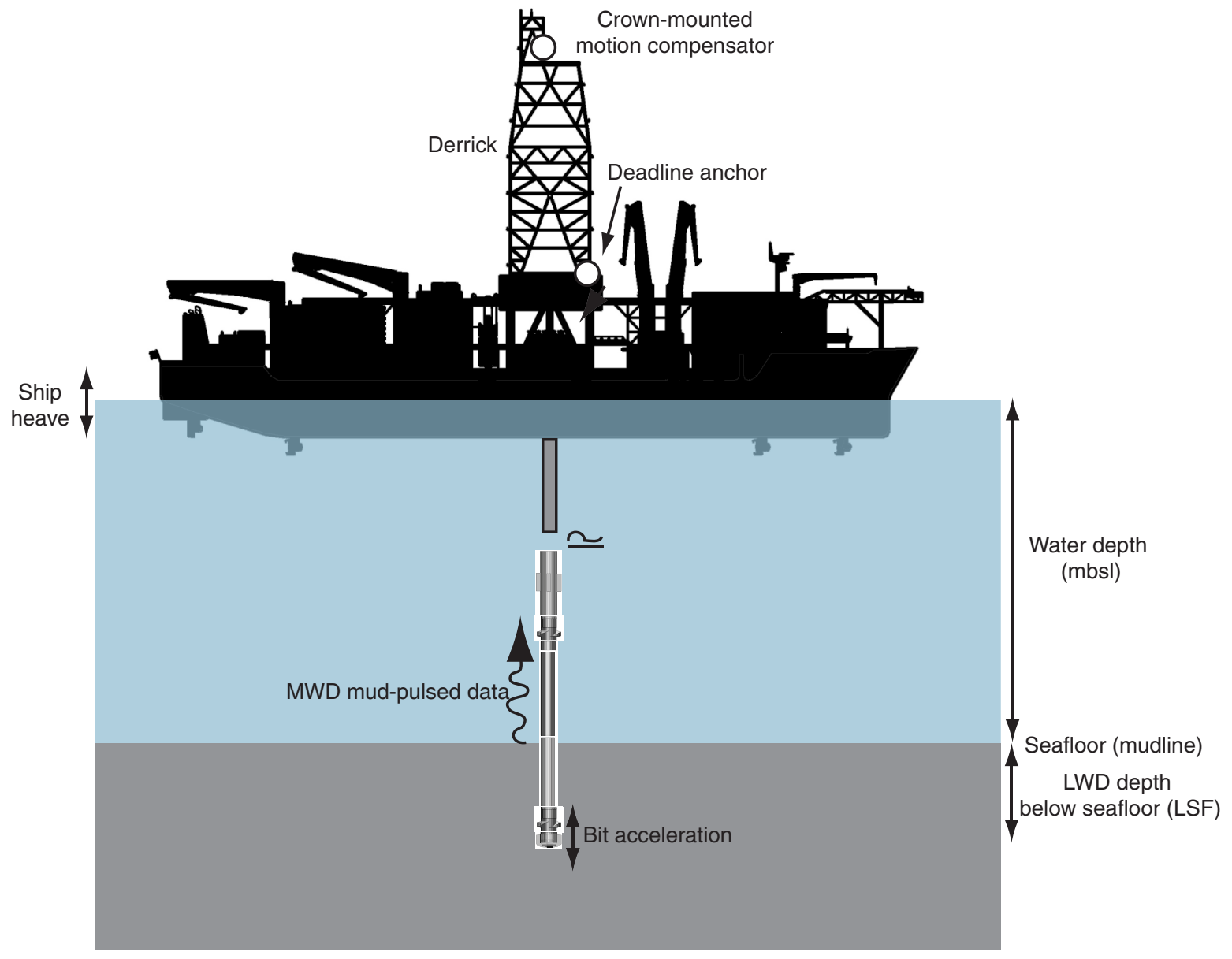


Figure F3. A. Schematic illustration of the MWD PowerPulse tool. (Continued on next page.)

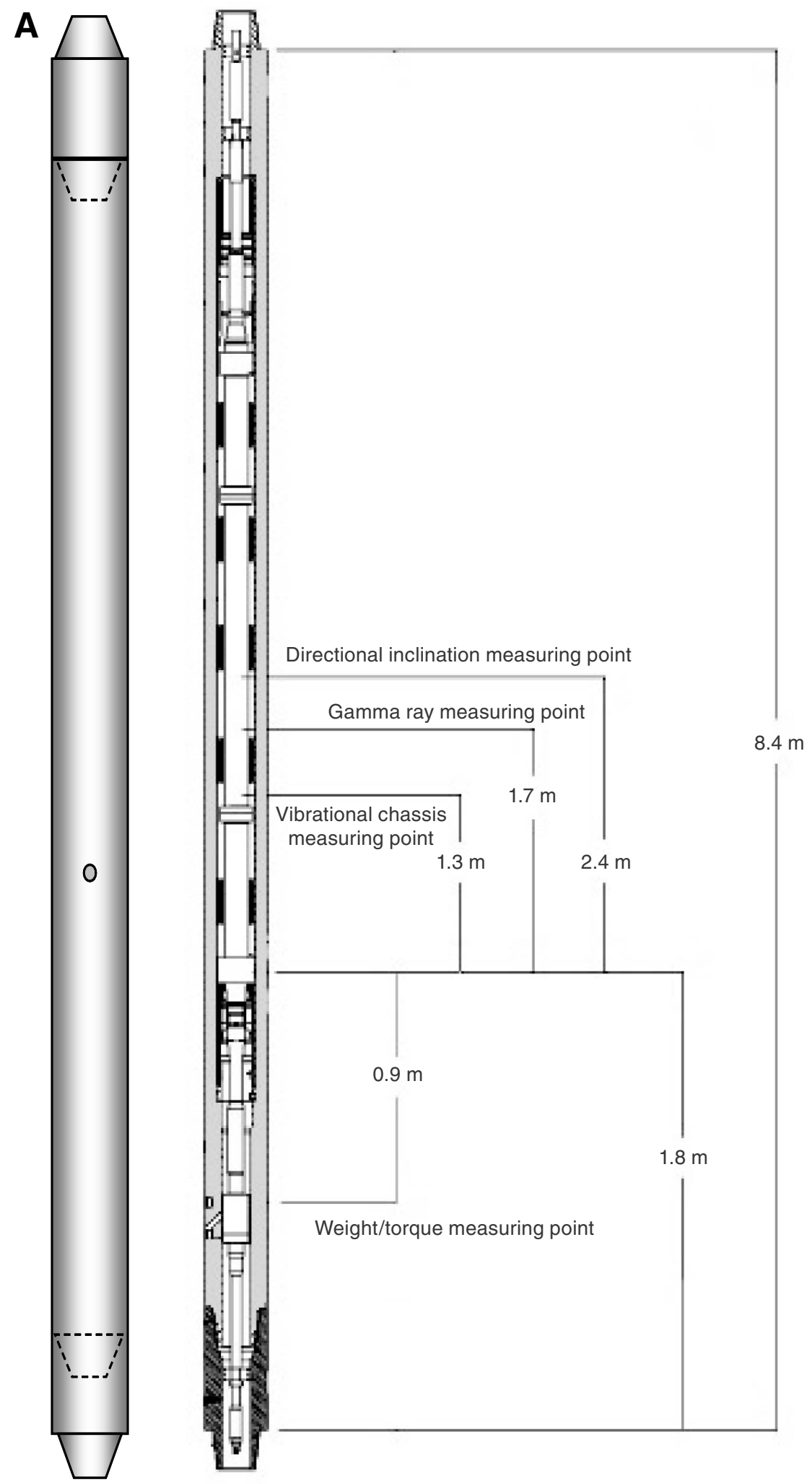


Figure F3 (continued). B. Configuration and principle of MWD fluid pulse telemetry.

B
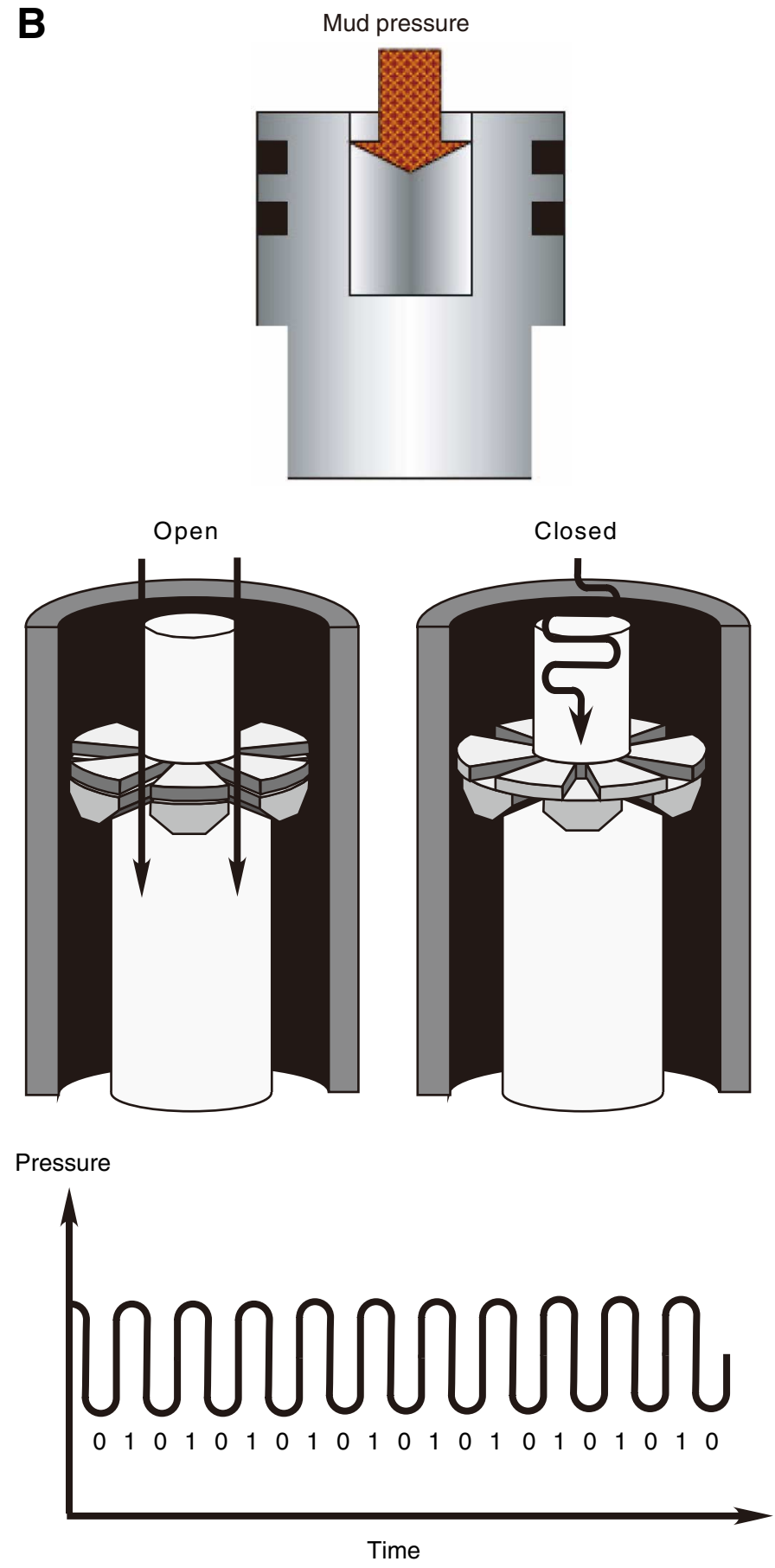
Figure F4. Position and components of the adnVISION tool.

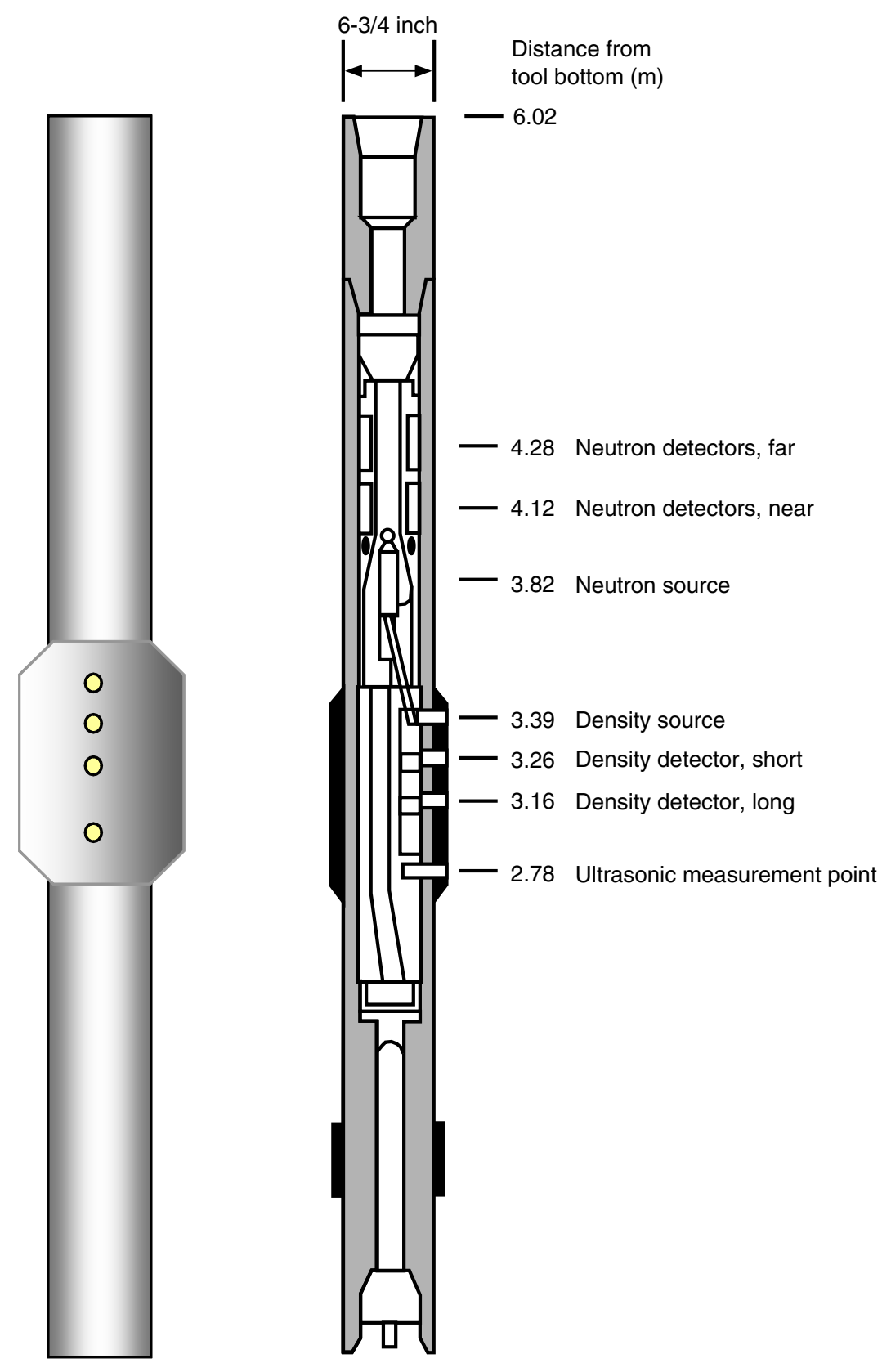


Figure F5. Position and components of the geoVISION tool.

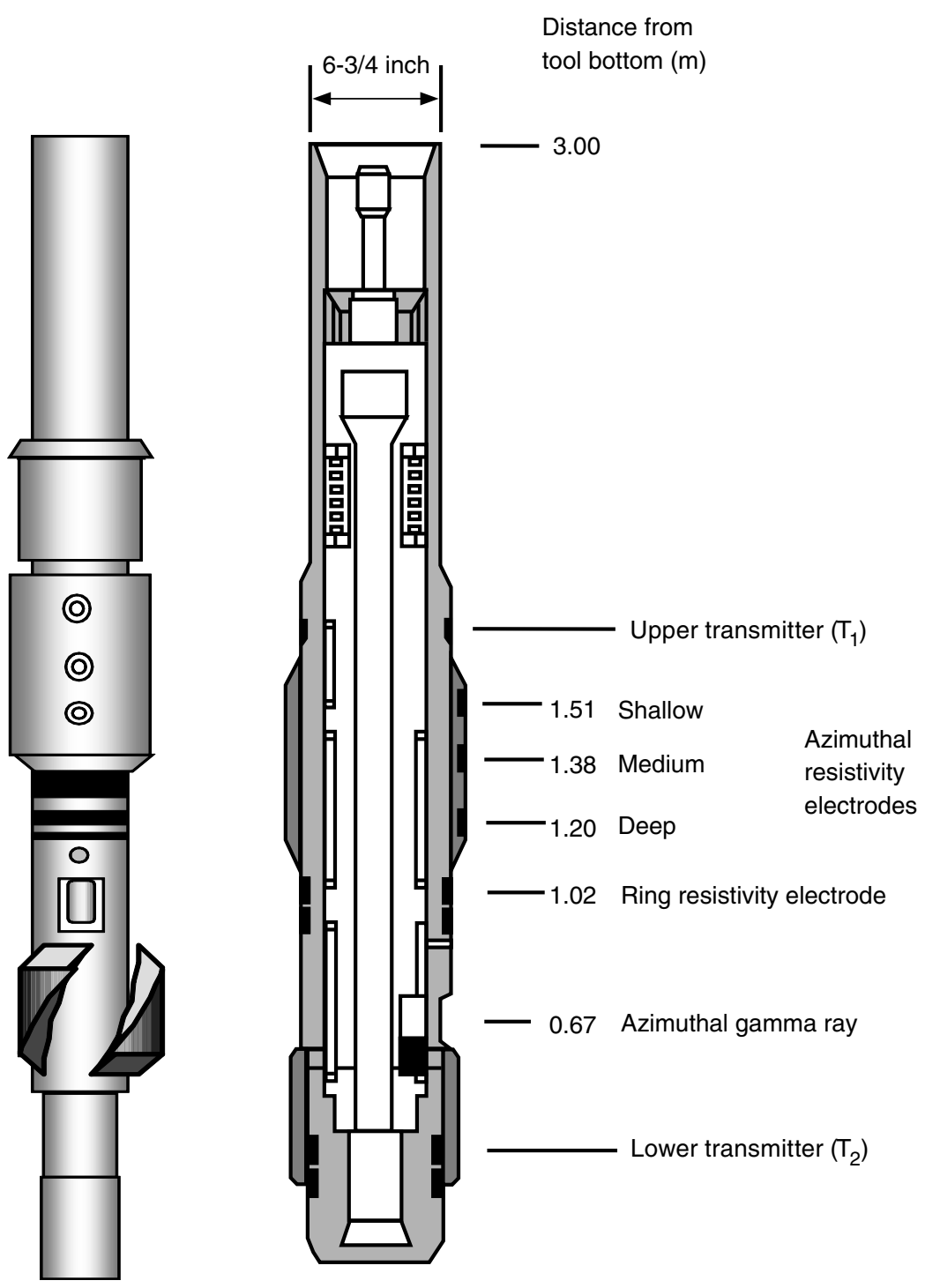


Figure F6. Position and components of the sonicVISION tool. ROP = rate of penetration.
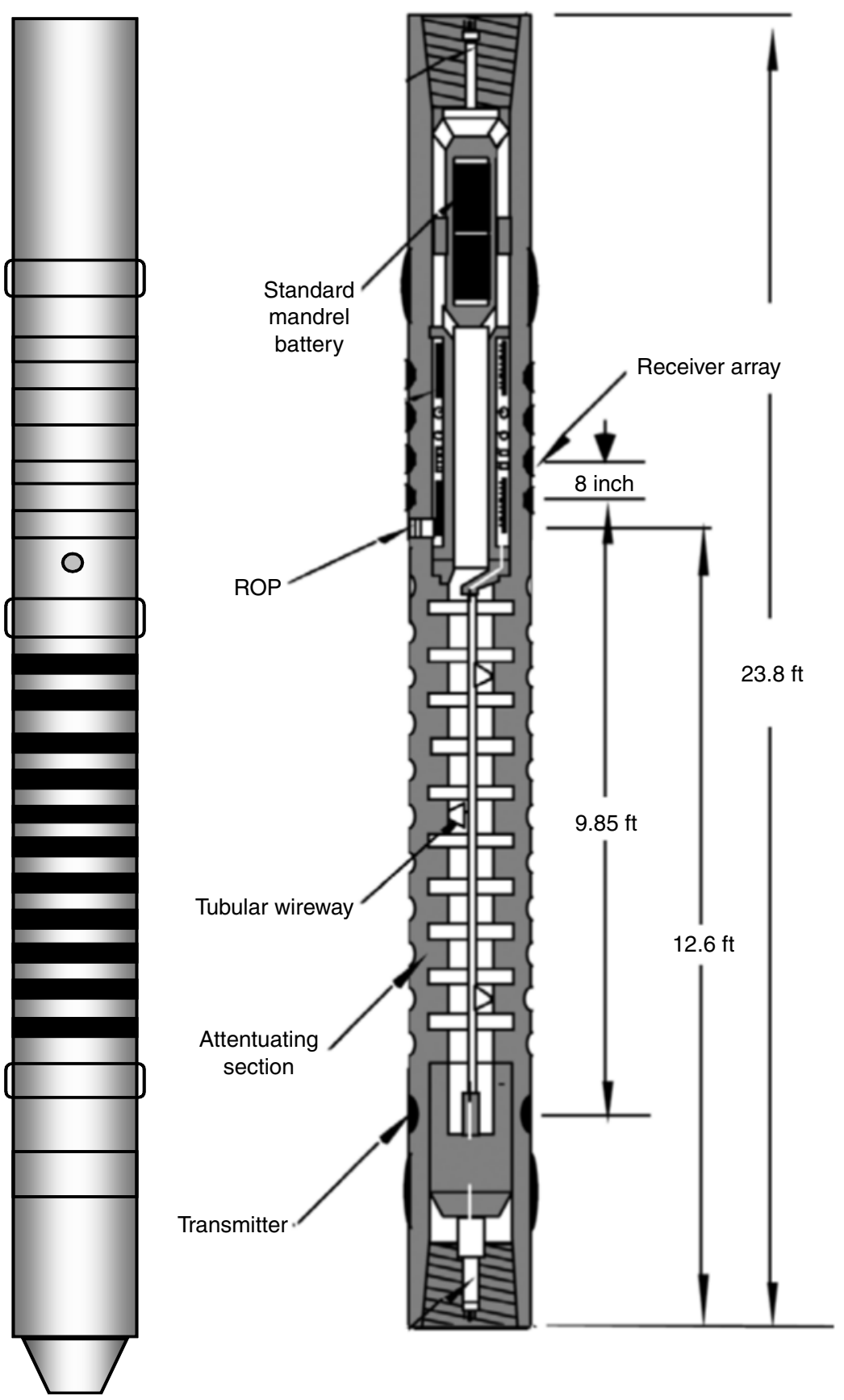
Figure F7. Position and components of the seismicVISION tool.

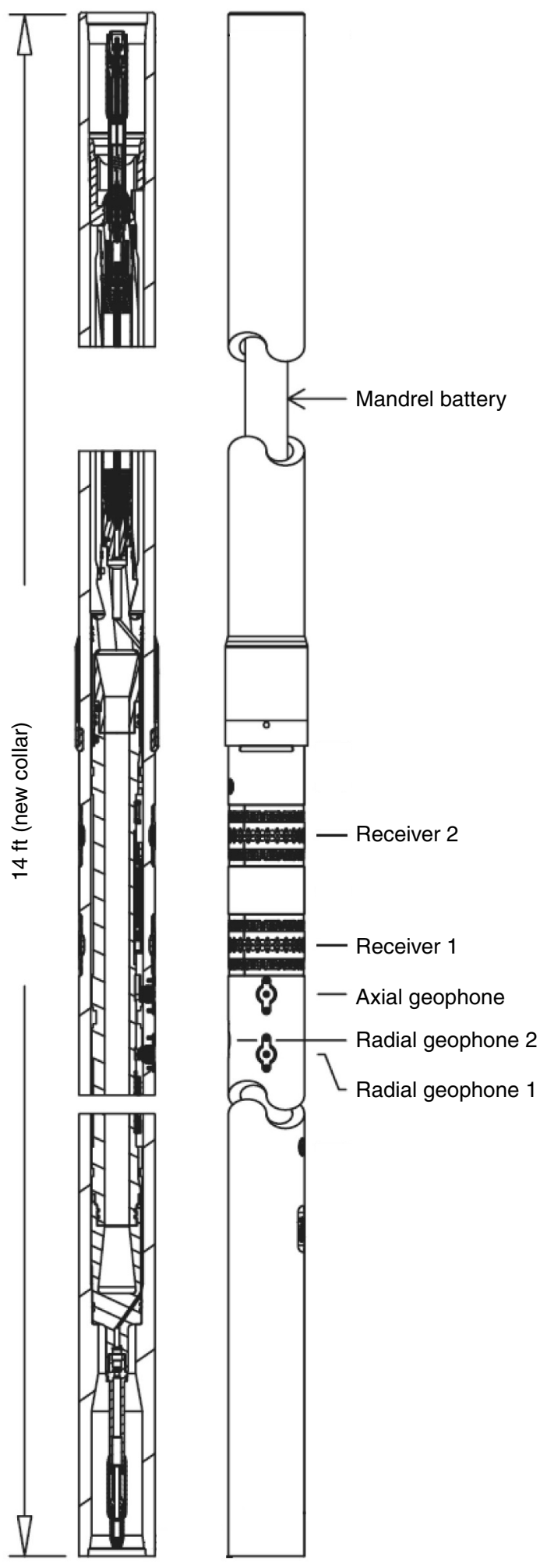


Figure F8. Shipboard structure and data flow. MWD = measurement while drilling, APWD = annular pressure while drilling, LWD = logging while drilling, IDEAL = integrated drilling evaluation and logging system (Schlumberger), ADN = Azimuthal Density Neutron tool (adnVISION), GVR = geoVISION resistivity tool, LAS $=\log$ ASCII standard format, DLIS = digital logging interchange standard format, SEGY = Society of Exploration Geophysicists standard format "Y," DCS = Data Consulting Service (Schlumberger).

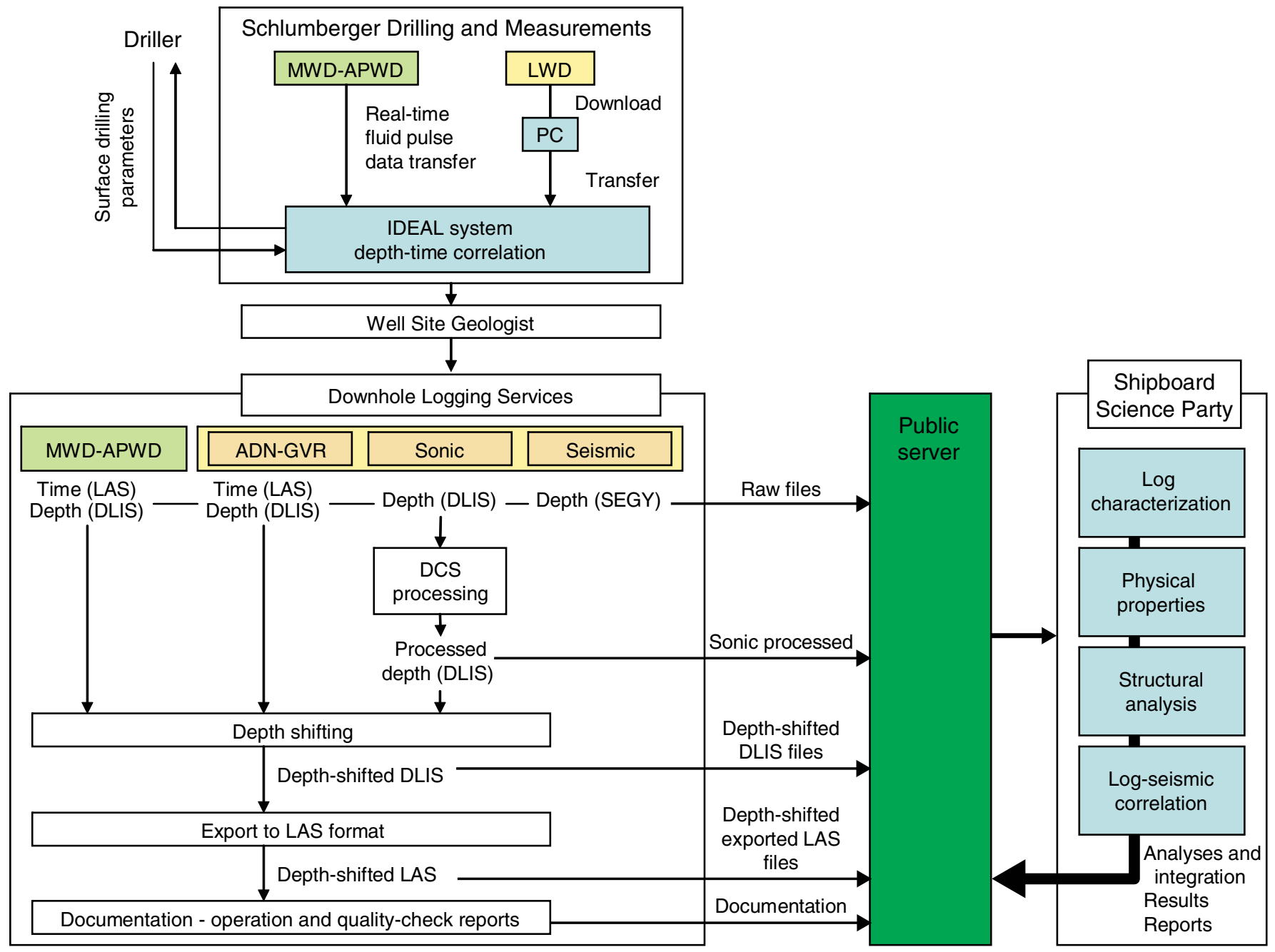


Figure F9. Presentation of data for operation description and quality assessment.
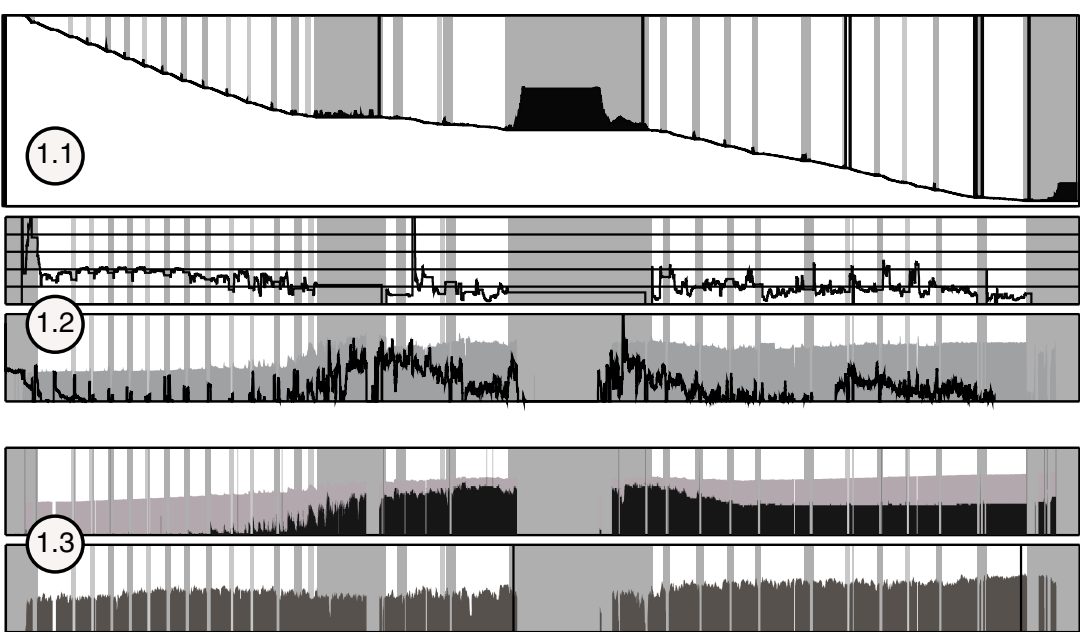

Time
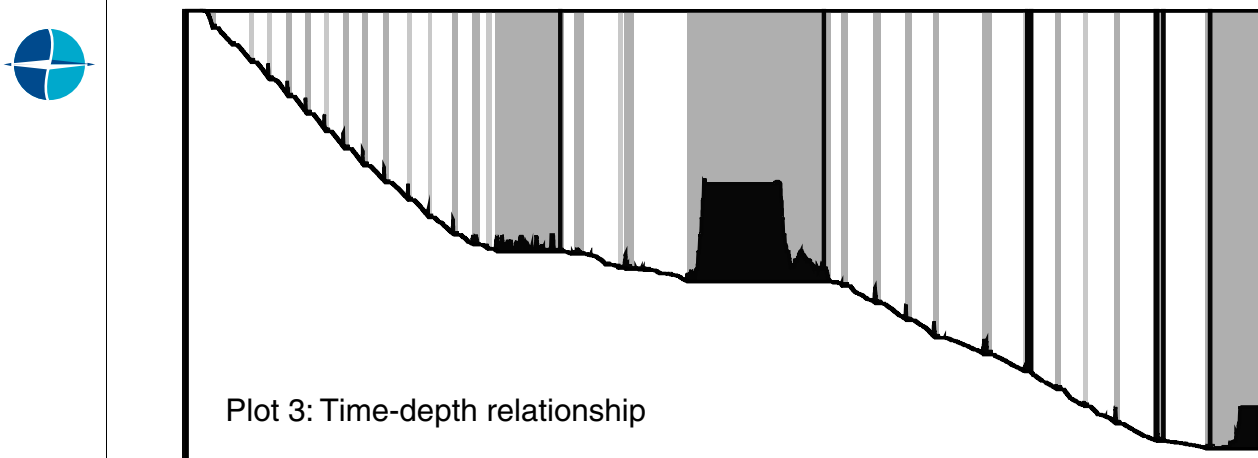

Main Plot 1: Time version

(1.1) Time-depth relationship

1.2 Rig floor and downhole drilling parameters

(1.3) Geophysical logs

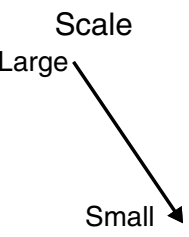

Main Plot 2: Depth version

(2.1) Time-depth relationship

(2.2) Rig floor and downhole drilling parameters

(2.3) Geophysical logs

(2.4) Sonic processing documentation

(2.5) Image quality assessment
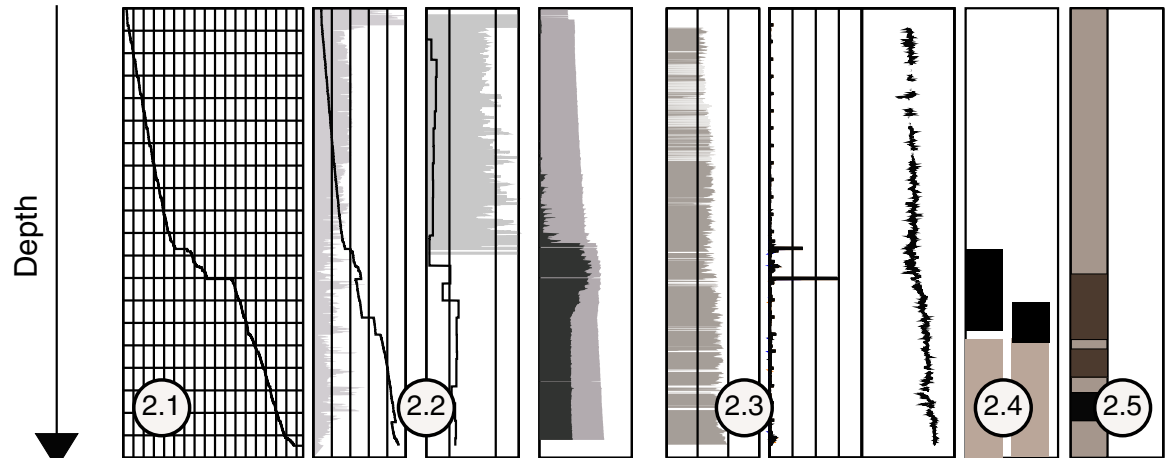
Figure F10. Stress polygons showing conditions of principal horizontal stresses favoring normal, strike-slip, and thrust faulting (Zoback et al., 2003). $S_{\mathrm{hmin}}=S_{\mathrm{H} \max }$ line is lower limit of possible stress states. Lines bounding the composite polygon on its upper and left sides are thresholds of failure; stress states to the left and above these lines cannot exist in the natural state. Different stress polygons apply for differing depths, pore pressures, and friction coefficients. $S_{\mathrm{hmin}}=$ minimum principal horizontal stress, $S_{\mathrm{H} \max }=$ maximum horizontal principal stress, $S_{\mathrm{v}}=$ vertical stress.

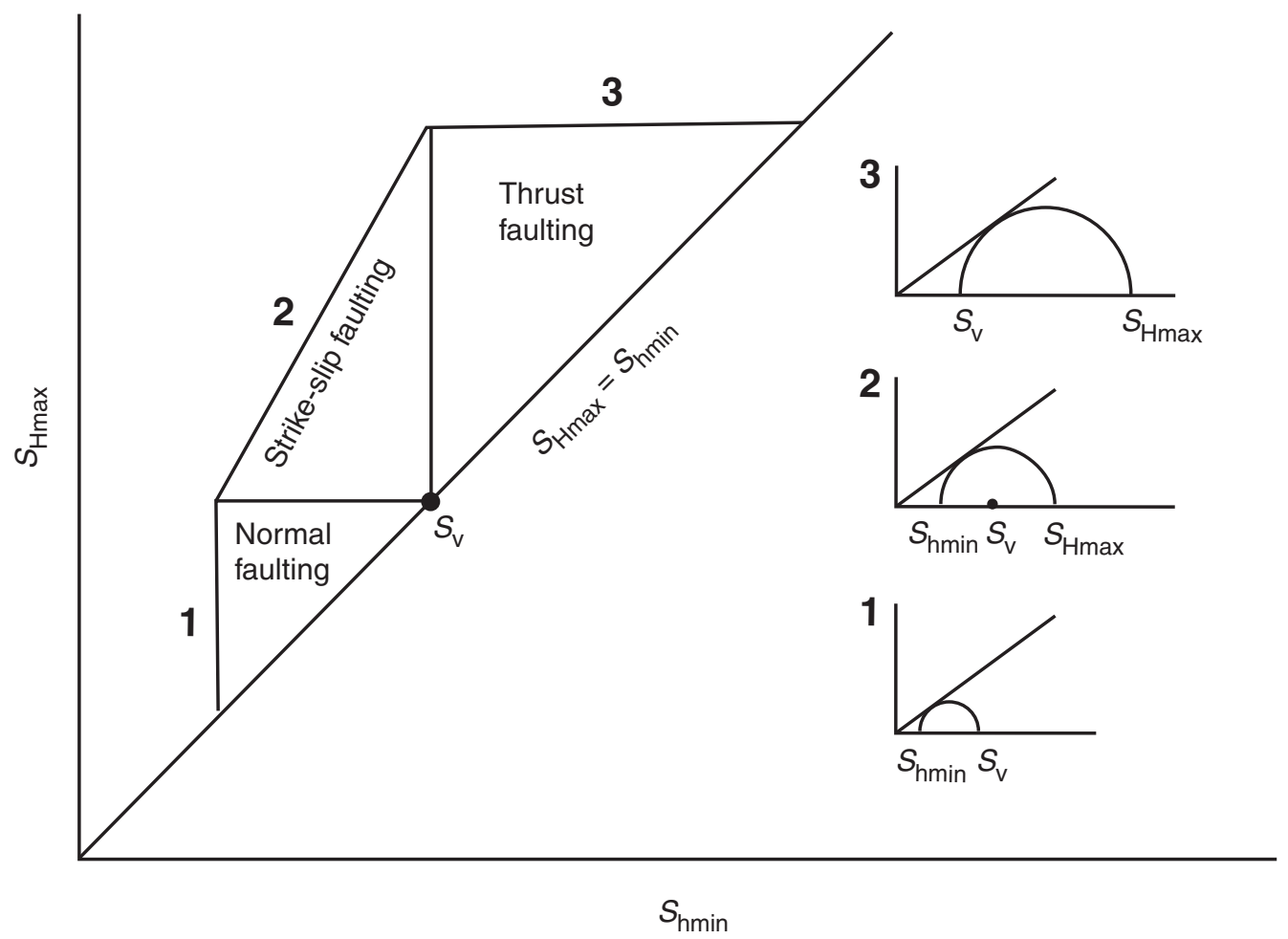


Figure F11. seismicVISION source array of three 250 inch $^{3}$ air guns.

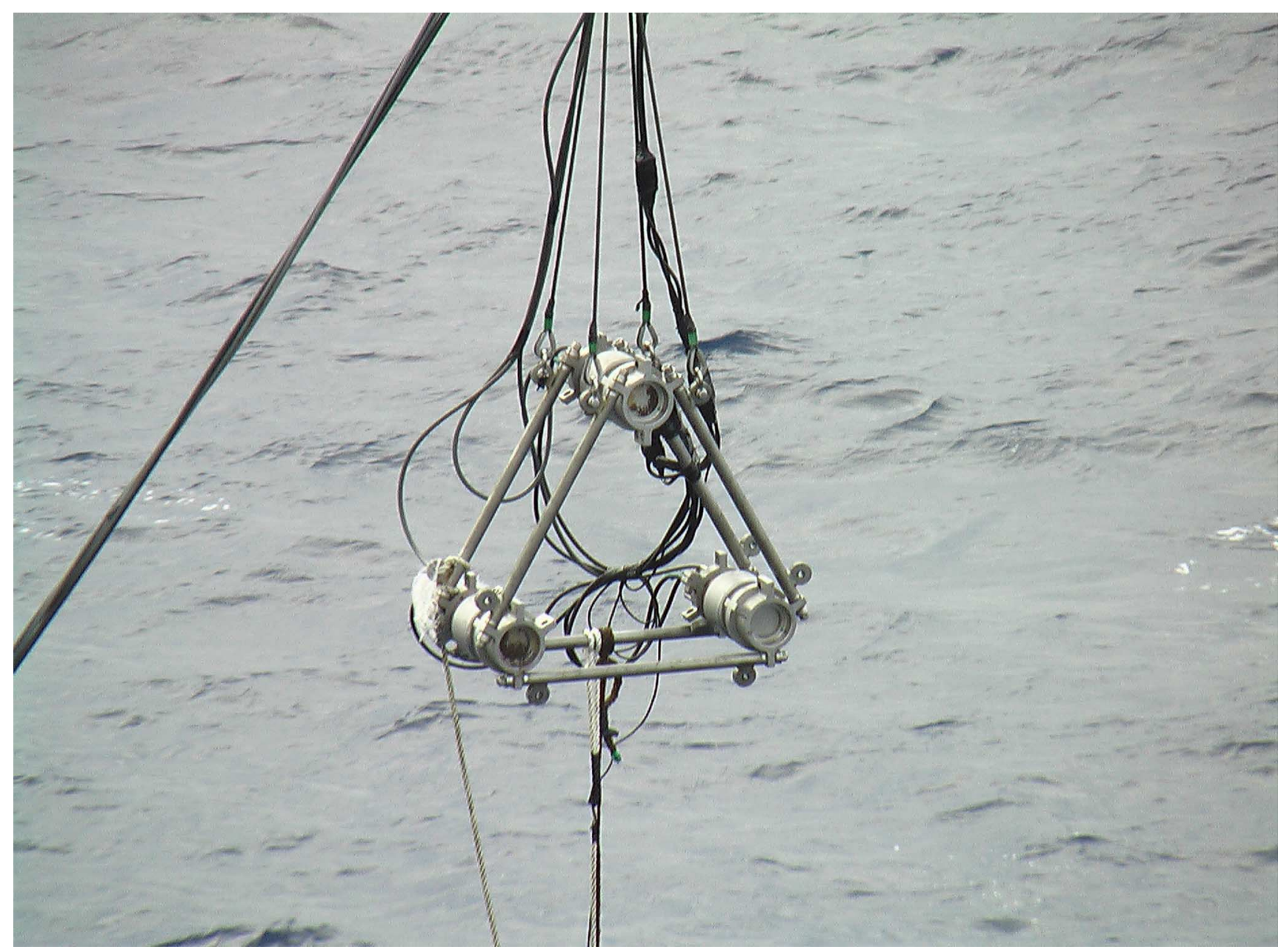


Figure F12. Deployment of the seismicVISION source array and acquisition geometry on the Chikyu.
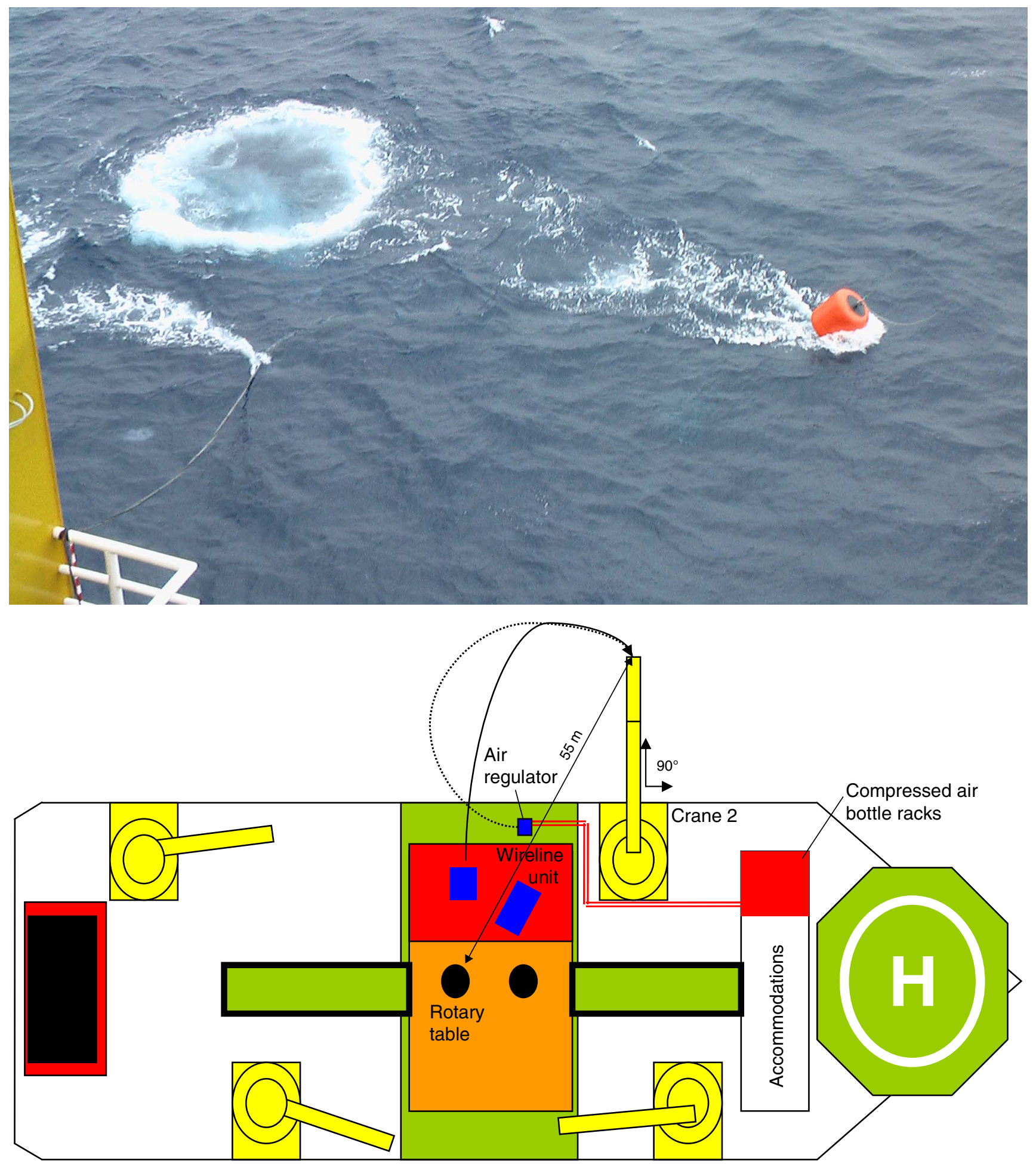
Figure F13. Firing locations for seismicVISION during drilling and drill pipe recovery. TD = total depth.

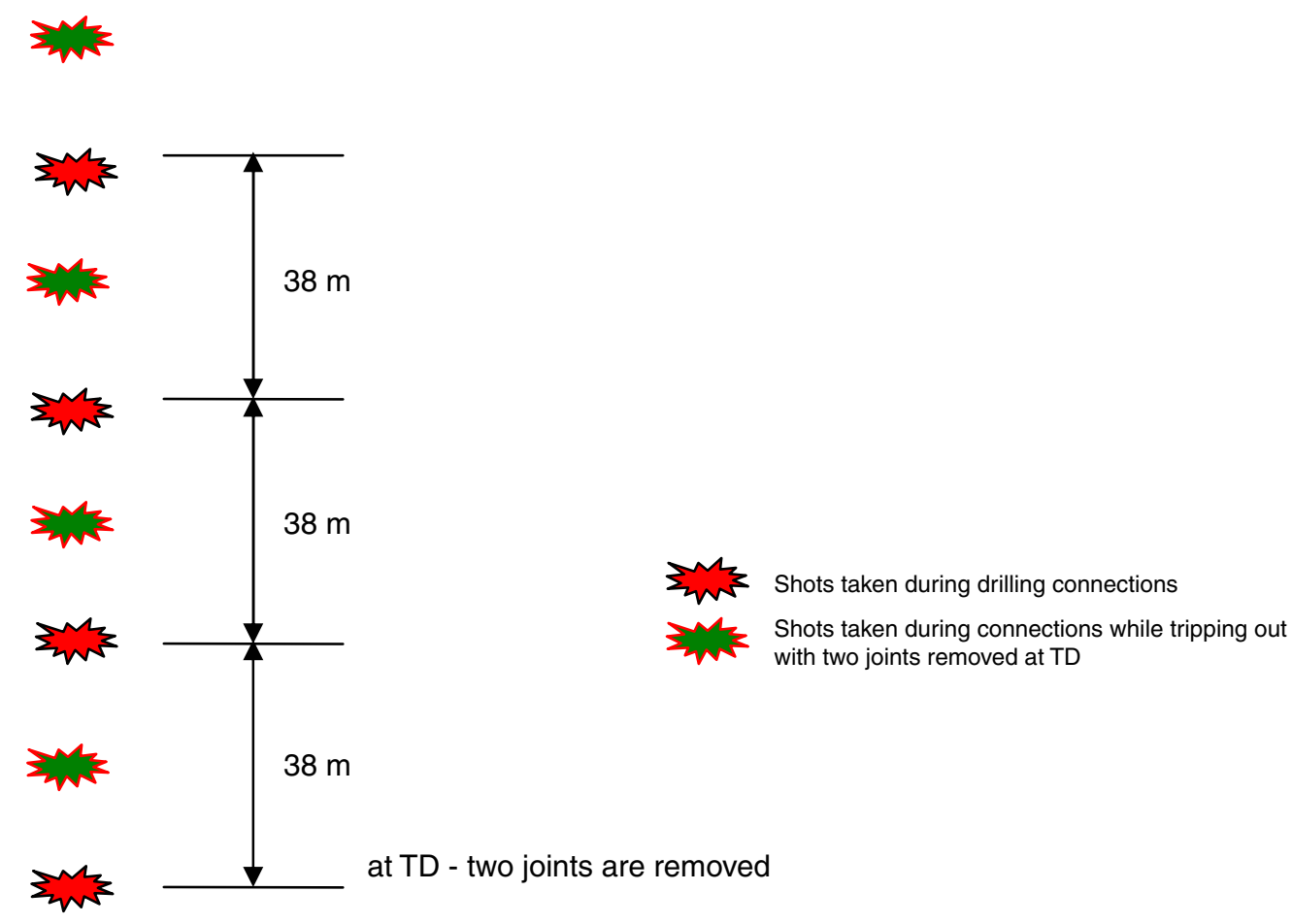


Table T1. MWD-APWD tools acronyms, descriptions, and units. (See table note.)

\begin{tabular}{|c|c|c|c|}
\hline Tool & Output & Description & Unit \\
\hline \multirow[t]{8}{*}{ MWD } & & Measurement-while-drilling tool & \\
\hline & HKLD & Hookload & kkgf \\
\hline & SWOB & Surface weight on bit & kkgf \\
\hline & SPPA & Standpipe pressure & $\mathrm{kPa}$ \\
\hline & $\mathrm{ROP} * 5$ & $5 \mathrm{ft}$ averaged rate of penetration & $\mathrm{m} / \mathrm{h}$ \\
\hline & GRM1 & LWD gamma ray & gAPI \\
\hline & CRPM_RT & Collar rotation & $\mathrm{rpm}$ \\
\hline & TRPM_RT & MWD turbine rotation speed & rpm \\
\hline \multirow[t]{10}{*}{ APWD } & & Annular-pressure-while-drilling tool & \\
\hline & APRS_MWD & Average annular pressure MWD & $\mathrm{kPa}$ \\
\hline & ATMP_MWD & Annular temperature MWD & ${ }^{\circ} \mathrm{C}$ \\
\hline & ECD_MWD & Equivalent circulating density & $\mathrm{g} / \mathrm{cm}^{3}$ \\
\hline & TUR_RPM & Turbine rotation & $\mathrm{rpm}$ \\
\hline & SHOCK_I & PowerPulse real-time shock counter & - \\
\hline & STICKNSLIP & Stick slip indicator & $\mathrm{cm} / \mathrm{min}$ \\
\hline & CRPM & Collar rotational speed & $\mathrm{cm} / \mathrm{min}$ \\
\hline & STUCK & Percent time stuck below $5 \mathrm{rpm}$ indicator & $\%$ \\
\hline & MTF & PowerPulse magnetic tool face angle & $\circ$ \\
\hline
\end{tabular}

Note: $L W D=$ logging while drilling, MWD = measurement while drilling.

Table T2. MWD-APWD-LWD real-time data acronyms, definitions, and units. (See table notes.)

\begin{tabular}{|c|c|c|c|}
\hline Tool & Output & Description & Unit \\
\hline \multirow[t]{6}{*}{ adnVISION } & & Azimuthal Density Neutron tool & \\
\hline & RHOB_DH_ADN_RT & Bulk density computed downhole & $\mathrm{g} / \mathrm{cm}^{3}$ \\
\hline & DRHO_DH_ADN_RT & Bulk density correction computed downhole & $\mathrm{g} / \mathrm{cm}^{3}$ \\
\hline & TNRA_ADN_RT & Thermal neutron ratio & - \\
\hline & TNPH_ADN_RT & Thermal neutron porosity & $\mathrm{pu}$ \\
\hline & ADIA_ADN_T & Average borehole diameter & inch \\
\hline \multirow{13}{*}{ geoVISION } & & geoVISION resistivity tool & \\
\hline & GR_RAB_RT & RAB gamma ray & gAPI \\
\hline & RES_RING_RT & Ring resistivity & $\Omega \mathrm{m}$ \\
\hline & RES_BIT_RT & Bit resistivity & $\Omega \mathrm{m}$ \\
\hline & RES_BS_RT & Shallow button resistivity & $\Omega \mathrm{m}$ \\
\hline & RES_BM_RT & Medium button resistivity & $\Omega \mathrm{m}$ \\
\hline & RES_BD_RT & Deep button resistivity & $\Omega \mathrm{m}$ \\
\hline & RB_RT & Relative bearing, real time & $\circ$ \\
\hline & HAZI_RT & Hole azimuth, real time & $\circ$ \\
\hline & DEVI_RT & Hole deviation, real time & $\circ$ \\
\hline & ECAL_RAB_RT & Electrical caliper computed from RAB resistivities & inch \\
\hline & SHK_RAB_TRANS_RT & Transverse shock & - \\
\hline & SHK_RAB_AX_RT & Axial shocks & - \\
\hline \multirow[t]{5}{*}{ sonicVISION } & & Sonic-while-drilling tool & \\
\hline & DTCO & $\Delta \mathrm{T}$ compressional & $\mu \mathrm{s} / \mathrm{ft}$ \\
\hline & $\mathrm{CHC}$ & Sonic compressional semblance & - \\
\hline & C_PEAK4_S & Four peaks with $\Delta \mathrm{T}, \mathrm{CO}$, and $\mathrm{TT}$ & - \\
\hline & SONSK_S & Shock risk & - \\
\hline \multirow[t]{3}{*}{ seismicVISION } & & Seismic-while-drilling tool & \\
\hline & A_SWAV_Z & SVWD generic Dpoint (utility frame) & - \\
\hline & SEISK_z & SVWD shock risk & - \\
\hline
\end{tabular}

Notes: $\mathrm{RAB}=$ resistivity-at-the-bit. $\mathrm{CO}=$ coherence, $\mathrm{TT}=$ traveltime. SVWD = seismicVISION while drilling tool. 
Table T3. LWD tool memory data acronyms, descriptions, and units. (See table notes.)

\begin{tabular}{|c|c|c|c|}
\hline Tool & Output & Description & Unit \\
\hline \multirow[t]{14}{*}{ adnVISION } & & Azimuthal Density Neutron tool & \\
\hline & ROMT & Density (rotationally processed) & $\mathrm{g} / \mathrm{cm}^{3}$ \\
\hline & RHOB & Density (average bulk in situ formation density) & $\mathrm{g} / \mathrm{cm}^{3}$ \\
\hline & DRHO & Bulk density correction & $\mathrm{g} / \mathrm{cm}^{3}$ \\
\hline & TAB_DEN & Density time after bit & $\mathrm{s}$ \\
\hline & $\mathrm{NRHO}$ & Bulk density, enhanced vertical resolution & $\mathrm{g} / \mathrm{cm}^{3}$ \\
\hline & DCAL & Differential caliper & inch \\
\hline & TNPH & Thermal neutron porosity (ratio method) in selected lithology & pu \\
\hline & TNPH_UNC & Hole size-corrected thermal neutron porosity & pu \\
\hline & PEF & Photoelectric factor & $\mathrm{b} / \mathrm{e}^{-}$ \\
\hline & TAB_NEU & Neutron time after bit & $\mathrm{s}$ \\
\hline & IDDR & Image-derived density correction & $\mathrm{g} / \mathrm{cm}^{3}$ \\
\hline & IDPE & Image-derived photoelectric factor & $\mathrm{b} / \mathrm{e}^{-}$ \\
\hline & IDRO & Image-derived density & \\
\hline \multirow[t]{18}{*}{ geoVISION } & & geoVISION resistivity tool & \\
\hline & GR & Gamma ray & gAPI \\
\hline & RES_RING & Ring resistivity & $\Omega \mathrm{m}$ \\
\hline & RES_BIT & Bit resistivity & $\Omega \mathrm{m}$ \\
\hline & RES_BS & Shallow button resistivity & $\Omega \mathrm{m}$ \\
\hline & RES_BM & Medium button resistivity & $\Omega \mathrm{m}$ \\
\hline & RES_BD & Deep button resistivity & $\Omega \mathrm{m}$ \\
\hline & RES_BS_IMG & Shallow button resistivity image & $\Omega \mathrm{m}$ \\
\hline & RES_BM_IMG & Medium button resistivity image & $\Omega \mathrm{m}$ \\
\hline & RES_BD_IMG & Deep button resistivity image & $\Omega \mathrm{m}$ \\
\hline & TAB_RAB_BS & Shallow button resistivity time after bit & s \\
\hline & TAB_RAB_BM & Medium button resistivity time after bit & $\mathrm{s}$ \\
\hline & TAB_RAB_BD & Deep button resistivity time after bit & $\mathrm{s}$ \\
\hline & ECAL_RAB & Electrical caliper computed from RAB resistivities & inch \\
\hline & HAZI & Hole azimuth relative to true north & $\circ$ \\
\hline & DEVI & Hole deviation from well survey & $\circ$ \\
\hline & P1NO & Pad 1 azimuth in plane orthogonal to tool axis & $\circ$ \\
\hline & TAB_RAB_RING & Ring resistivity time after bit & $\mathrm{s}$ \\
\hline \multirow[t]{14}{*}{ sonicVISION } & & Sonic-while-drilling tool & \\
\hline & SPWi & Receiver_i spectrum array & - \\
\hline & Wfi & Filtered waveform_i, $0.5 \mathrm{ft}$ averaged & \\
\hline & WFiT & Filtered waveform_i, 2 inch averaged & \\
\hline & DTTA & $\Delta \mathrm{T}$ compressional from transmitter array & $\mu \mathrm{s} / \mathrm{ft}$ \\
\hline & DTRA & $\Delta \mathrm{T}$ compressional from receiver array & $\mu \mathrm{s} / \mathrm{ft}$ \\
\hline & DTBC & $\Delta \mathrm{T}$ compressional borehole compensated & $\mu \mathrm{s} / \mathrm{ft}$ \\
\hline & DTDF & $\Delta \mathrm{T}$ compressional difference between DTRA and DTTA & $\mu \mathrm{s} / \mathrm{ft}$ \\
\hline & CHRA & Coherence at compressional peak; receiver array & - \\
\hline & CHTA & Coherence at compressional peak; transmitter array & - \\
\hline & TTRA & Transit time at compressional peak; receiver array & $\mu \mathrm{s}$ \\
\hline & TTTA & Transit time at compressional peak; transmitter array & $\mu \mathrm{s}$ \\
\hline & ITTI & Integrated transit time & $\mathrm{ms}$ \\
\hline & DTCO & $\Delta \mathrm{T}$ compressional & $\mu \mathrm{s} / \mathrm{ft}$ \\
\hline \multirow[t]{6}{*}{ seismicVISION } & & Seismic-while-drilling tool & \\
\hline & SVWD_001_nascent_shot_s1.segy & Raw shots of surface hydrophone & \\
\hline & SVWD_001_nascent_shot_h.segy & Raw shots of downhole hydrophone & \\
\hline & SVWD_001_nascent_shot_x.segy & Raw shots of downhole geophone; $x$ & \\
\hline & SVWD_001_nascent_shot_y.segy & Raw shots of downhole geophone; $y$ & \\
\hline & SVWD_001_nascent_shot_z.segy & Raw shots of downhole geophone; $z$ & \\
\hline
\end{tabular}

Notes: $\mathrm{i}=$ receiver number $(1,2,3$, or 4$) . \mathrm{RAB}=$ resistivity-at-the-bit. $\mathrm{DTRA}=\Delta \mathrm{T}$ compressional from receiver array, $\mathrm{DTTA}=\Delta \mathrm{T}$ compressional from transmitter array. 
Table T4. Fluid pulse telemetry frame. (See table notes.)

\begin{tabular}{|c|c|c|c|c|c|c|c|c|}
\hline \multirow[b]{2}{*}{ Tool } & \multirow[b]{2}{*}{ D_Point } & \multirow[b]{2}{*}{ Unit } & \multirow[b]{2}{*}{ Description } & \multirow[b]{2}{*}{ Bits } & \multirow[b]{2}{*}{$\begin{array}{l}\text { Update } \\
\text { rate (s) }\end{array}$} & \multicolumn{3}{|c|}{ Vertical sampling $(\mathrm{m})$} \\
\hline & & & & & & $\begin{array}{c}\mathrm{ROP} \\
10 \mathrm{~m} / \mathrm{h}\end{array}$ & $\begin{array}{c}\text { ROP } \\
25 \mathrm{~m} / \mathrm{h}\end{array}$ & $\begin{array}{c}\mathrm{ROP} \\
45 \mathrm{~m} / \mathrm{h}\end{array}$ \\
\hline SVWD & A_SWAV_z & - & SVWD generic Dpoint (utility frame) & 8 & 7 & 0.02 & 0.05 & 0.09 \\
\hline PP & APRS & psi & Average annular pressure & 15 & 23.3 & 0.06 & 0.16 & 0.29 \\
\hline RAB & GRRA_r & gAPI & Average gamma ray & 8 & 35 & 0.10 & 0.24 & 0.44 \\
\hline sonic & C_PEAK4_S & - & Four peaks with $\Delta \mathrm{T}, \mathrm{CO}$, and $\mathrm{TT}$ & 9 & 11.7 & 0.03 & 0.08 & 0.15 \\
\hline SVWD & A_SWAV_Z & - & SVWD generic Dpoint (utility frame) & 8 & 7 & 0.02 & 0.05 & 0.09 \\
\hline RAB & RING_r & $\Omega \mathrm{m}$ & Ring resistivity & 10 & 35 & 0.10 & 0.24 & 0.44 \\
\hline RAB & RBIT_r & $\Omega \mathrm{m}$ & Bit resistivity & 10 & 35 & 0.10 & 0.24 & 0.44 \\
\hline RAB & RDBA_ $r$ & $\Omega \mathrm{m}$ & Average deep button resistivity & 10 & 35 & 0.10 & 0.24 & 0.44 \\
\hline SVWD & A_SWAV_Z & - & SVWD generic Dpoint (utility frame) & 8 & 7 & 0.02 & 0.05 & 0.09 \\
\hline sonic & DTCO_s & $\mu \mathrm{s} / \mathrm{ft}$ & Sonic compressional $\Delta \mathrm{T}$ & 10 & 35 & 0.10 & 0.24 & 0.44 \\
\hline sonic & CHCO_S & - & Sonic compressional semblance & 7 & 35 & 0.10 & 0.24 & 0.44 \\
\hline sonic & C_PEAK4_s & - & Four peaks with $\Delta \mathrm{T} C O$, and $\mathrm{TT}$ & 9 & 11.7 & 0.03 & 0.08 & 0.15 \\
\hline ADN & RHOB_a & $\mathrm{g} / \mathrm{cm}^{3}$ & Average bulk density ( $\rho$ ) & 9 & 35 & 0.10 & 0.24 & 0.44 \\
\hline ADN & DRHO a & $\mathrm{g} / \mathrm{cm}^{3}$ & Total delta density $(\rho)$ & 8 & 35 & 0.10 & 0.24 & 0.44 \\
\hline SVWD & A_SWAV_Z & - & SVWD generic Dpoint (utility frame) & 8 & 7 & 0.02 & 0.05 & 0.09 \\
\hline ADN & TNRA_a & - & Total neutron ratio & 9 & 35 & 0.10 & 0.24 & 0.44 \\
\hline PP & APRS & psi & Average annular pressure & 15 & 23.3 & 0.06 & 0.16 & 0.29 \\
\hline ADN & ADIA_a & & & 8 & 35 & 0.10 & 0.24 & 0.44 \\
\hline sonic & C_PEAK4_s & - & Four peaks with $\Delta \mathrm{T}, \mathrm{CO}$, and $\mathrm{TT}$ & 9 & 11.7 & 0.03 & 0.08 & 0.15 \\
\hline SVWD & A_SWAV_Z & - & SVWD generic Dpoint (utility frame) & 8 & 7 & 0.02 & 0.05 & 0.09 \\
\hline $\mathrm{PP}$ & ATMP & ${ }^{\circ} \mathrm{C}$ & Annulus temperature & 8 & 70 & 0.19 & 0.49 & 0.88 \\
\hline PP & TUR_RPM & $\mathrm{rpm}$ & Turbine rotation & 7 & 70 & 0.19 & 0.49 & 0.88 \\
\hline RAB & SHKÄr $r$ & - & Axial shock risk & 2 & 70 & 0.19 & 0.49 & 0.88 \\
\hline RAB & SHKT_r & - & Transverse shock risk & 2 & 70 & 0.19 & 0.49 & 0.88 \\
\hline sonic & SONSK_S & - & Shock risk & 2 & 70 & 0.19 & 0.49 & 0.88 \\
\hline ADN & ADNSK_a & - & Shock risk & 2 & 70 & 0.19 & 0.49 & 0.88 \\
\hline PP & SHOCK_I & - & PowerPulse real-time shock counter & 8 & 70 & 0.19 & 0.49 & 0.88 \\
\hline SWWD & A SWAV $z$ & - & SVWD generic Dpoint (utility frame) & 8 & 7 & 0.02 & 0.05 & 0.09 \\
\hline SVWD & SEISK_z & - & SVWD shock risk & 2 & 70 & 0.19 & 0.49 & 0.88 \\
\hline RAB & GRRA_r & gAPI & Average gamma ray & 8 & 35 & 0.10 & 0.24 & 0.44 \\
\hline sonic & C_PEAK4_s & - & Four peaks with $\Delta \mathrm{T}, \mathrm{CO}$, and $\mathrm{TT}$ & 9 & 11.7 & 0.03 & 0.08 & 0.15 \\
\hline RAB & RBIT_r & $\Omega \mathrm{m}$ & Bit resistivity & 10 & 35 & 0.10 & 0.24 & 0.44 \\
\hline SVWD & A_SWAV_z & - & SVWD generic Dpoint (utility frame) & 8 & 7 & 0.02 & 0.05 & 0.09 \\
\hline RAB & RING_r & $\Omega \mathrm{m}$ & Ring resistivity & 10 & 35 & 0.10 & 0.24 & 0.44 \\
\hline RAB & RDBA $r$ & $\Omega \mathrm{m}$ & Average deep button resistivity & 10 & 35 & 0.10 & 0.24 & 0.44 \\
\hline PP & APRS & psi & Average annular pressure & 15 & 23.3 & 0.06 & 0.16 & 0.29 \\
\hline SVWD & A_SWAV_Z & - & SVWD generic Dpoint (utility frame) & 8 & 7 & 0.02 & 0.05 & 0.09 \\
\hline sonic & C_PEAK4_s & - & Four peaks with $\Delta \mathrm{T}, \mathrm{CO}$, and $\mathrm{TT}$ & 9 & 11.7 & 0.03 & 0.08 & 0.15 \\
\hline sonic & DTCO_s & $\mu \mathrm{s} / \mathrm{ft}$ & Sonic compressional $\Delta \mathrm{T}$ & 10 & 35 & 0.10 & 0.24 & 0.44 \\
\hline sonic & CHCO_s & - & Sonic compressional semblance & 7 & 35 & 0.10 & 0.24 & 0.44 \\
\hline ADN & RHOB_a & $\mathrm{g} / \mathrm{cm}^{3}$ & Average bulk density ( $\rho$ ) & 9 & 35 & 0.10 & 0.24 & 0.44 \\
\hline SWWD & A SWAV $z$ & - & SVWD generic Dpoint (utility frame) & 8 & 7 & 0.02 & 0.05 & 0.09 \\
\hline ADN & DRHO_a & $\mathrm{g} / \mathrm{cm}^{3}$ & Total delta density $(\rho)$ & 8 & 35 & 0.10 & 0.24 & 0.44 \\
\hline ADN & TNRA_a & - & Total neutron ratio & 9 & 35 & 0.10 & 0.24 & 0.44 \\
\hline PP & STICKNSLIP & $\mathrm{cm} / \mathrm{min}$ & Peak-peak collar rpm variations & 7 & 70 & 0.19 & 0.49 & 0.88 \\
\hline ADN & ADIA_a & inch & Average hole diameter (caliper) & 8 & 35 & 0.10 & 0.24 & 0.44 \\
\hline sonic & C_PEAK4_S & - & Four peaks with $\Delta \mathrm{T}, \mathrm{CO}$, and $\mathrm{TT}$ & 9 & 11.7 & 0.03 & 0.08 & 0.15 \\
\hline SVWD & A_SWAV_Z & - & SVWD generic Dpoint (utility frame) & 8 & 7 & 0.02 & 0.05 & 0.09 \\
\hline PP & CRPM & $\mathrm{cm} / \mathrm{min}$ & Average MWD collar rpm & 8 & 70 & 0.19 & 0.49 & 0.88 \\
\hline PP & STUCK & - & Percent time stuck & 2 & 70 & 0.19 & 0.49 & 0.88 \\
\hline
\end{tabular}

Notes: Used while the tool is rotating. Fluid pulse telemetry frame while rotating $=$ update rate at $6.0 \mathrm{bps}$. ROP $=$ rate of penetration $(\mathrm{m} / \mathrm{h})$. SVWD = seismicVISION while drilling tool, PP = PowerPulse MWD tool, RAB = resistivity-at-the-bit, sonic = sonicVISION while drilling tool, $\mathrm{ADN}=$ Azimuthal Density Neutron tool (adnVISION). $\mathrm{CO}=$ coherence, $\mathrm{TT}=$ traveltime. 
Table T5. Fluid pulse telemetry frame. (See table notes.)

\begin{tabular}{|c|c|c|c|c|c|c|c|c|}
\hline \multirow[b]{2}{*}{ Tool } & \multirow[b]{2}{*}{ D_Point } & \multirow[b]{2}{*}{ Unit } & \multirow[b]{2}{*}{ Description } & \multirow[b]{2}{*}{ Bits } & \multirow[b]{2}{*}{$\begin{array}{l}\text { Update } \\
\text { rate (s) }\end{array}$} & \multicolumn{3}{|c|}{ Vertical sampling $(\mathrm{m})$} \\
\hline & & & & & & $\begin{array}{c}\text { ROP } \\
10 \mathrm{~m} / \mathrm{h}\end{array}$ & $\begin{array}{c}\text { ROP } \\
25 \mathrm{~m} / \mathrm{h}\end{array}$ & $\begin{array}{c}\text { ROP } \\
45 \mathrm{~m} / \mathrm{h}\end{array}$ \\
\hline PP & MTFS & $\circ$ & PowerPulse magnetic tool face angle & 6 & 68.2 & 0.19 & 0.47 & 0.85 \\
\hline PP & APRS & psi & Average annular pressure & 15 & 22.7 & 0.06 & 0.16 & 0.28 \\
\hline GVR & GRRA_r & gAPI & Average gamma ray & 8 & 34.1 & 0.09 & 0.24 & 0.43 \\
\hline SVWD & A_SWAV_Z & - & SVWD generic Dpoint (utility frame) & 8 & 6.8 & 0.02 & 0.05 & 0.09 \\
\hline sonic & C_PEAK4_S & - & Four peaks with $\Delta \mathrm{T}, \mathrm{CO}$, and $\mathrm{TT}$ & 9 & 11.4 & 0.03 & 0.08 & 0.14 \\
\hline GVR & RING_r & $\Omega \mathrm{m}$ & Ring resistivity & 10 & 34.1 & 0.09 & 0.24 & 0.43 \\
\hline GVR & RBIT_r & $\Omega \mathrm{m}$ & Bit resistivity & 10 & 34.1 & 0.09 & 0.24 & 0.43 \\
\hline SVWD & A_SWAV_Z & - & SVWD generic Dpoint (utility frame) & 8 & 6.8 & 0.02 & 0.05 & 0.09 \\
\hline GVR & RDBA_r & $\Omega \mathrm{m}$ & Average deep button resistivity & 10 & 34.1 & 0.09 & 0.24 & 0.43 \\
\hline sonic & DTCO_s & $\mu \mathrm{s} / \mathrm{ft}$ & Sonic compressional $\Delta \mathrm{T}$ & 10 & 34.1 & 0.09 & 0.24 & 0.43 \\
\hline sonic & CHCO_s & - & Sonic compressional semblance & 7 & 34.1 & 0.09 & 0.24 & 0.43 \\
\hline sonic & C_PEAK4_S & - & Four peaks with $\Delta \mathrm{T}, \mathrm{CO}$, and $\mathrm{TT}$ & 9 & 11.4 & 0.03 & 0.08 & 0.14 \\
\hline SVWD & A_SWAV_Z & - & SVWD generic Dpoint (utility frame) & 8 & 6.8 & 0.02 & 0.05 & 0.09 \\
\hline ADN & RHOB_a & $\mathrm{g} / \mathrm{cm}^{3}$ & Average bulk density $(\rho)$ & 9 & 34.1 & 0.09 & 0.24 & 0.43 \\
\hline ADN & DRHO_a & $\mathrm{g} / \mathrm{cm}^{3}$ & Total delta density $(\rho)$ & 8 & 34.1 & 0.09 & 0.24 & 0.43 \\
\hline ADN & TNRA_a & - & Total neutron ratio & 9 & 34.1 & 0.09 & 0.24 & 0.43 \\
\hline SVWD & A_SWAV_Z & - & SVWD generic Dpoint (utility frame) & 8 & 6.8 & 0.02 & 0.05 & 0.09 \\
\hline PP & APRS & psi & Annulus pressure & 15 & 22.7 & 0.06 & 0.16 & 0.28 \\
\hline sonic & C_PEAK4_S & - & Four peaks with $\Delta \mathrm{T}, \mathrm{CO}$, and $\mathrm{TT}$ & 9 & 11.4 & 0.03 & 0.08 & 0.14 \\
\hline ADN & ADIA_a & inch & Average hole diameter (caliper) & 8 & 34.1 & 0.09 & 0.24 & 0.43 \\
\hline SVWD & A_SWAV_Z & - & SVWD generic Dpoint (utility frame) & 8 & 6.8 & 0.02 & 0.05 & 0.09 \\
\hline PP & ATMP & ${ }^{\circ} \mathrm{C}$ & Annular temperature & 8 & 68.2 & 0.19 & 0.47 & 0.85 \\
\hline PP & TUR_RPM & $\mathrm{rpm}$ & Turbine rotation & 7 & 68.2 & 0.19 & 0.47 & 0.85 \\
\hline GVR & SHKÄ_r & - & Axial shock risk & 2 & 68.2 & 0.19 & 0.47 & 0.85 \\
\hline GVR & SHKT_r & - & Transverse shock risk & 2 & 68.2 & 0.19 & 0.47 & 0.85 \\
\hline sonic & SONSK_S & - & Shock risk & 2 & 68.2 & 0.19 & 0.47 & 0.85 \\
\hline ADN & ADNSK_a & - & Shock risk & 2 & 68.2 & 0.19 & 0.47 & 0.85 \\
\hline PP & SHOCK_I & - & PowerPulse real-time shock counter & 8 & 68.2 & 0.19 & 0.47 & 0.85 \\
\hline SVWD & SEISK_Z & - & SVWD shock risk & 2 & 68.2 & 0.19 & 0.47 & 0.85 \\
\hline SVWD & A_SWAV_Z & - & SVWD generic Dpoint (utility frame) & 8 & 6.8 & 0.02 & 0.05 & 0.09 \\
\hline sonic & C_PEAK4_S & - & Four peaks with $\Delta \mathrm{T}, \mathrm{CO}$, and $\mathrm{TT}$ & 9 & 11.4 & 0.03 & 0.08 & 0.14 \\
\hline GVR & GRRA_r & gAPI & Average gamma ray & 8 & 34.1 & 0.09 & 0.24 & 0.43 \\
\hline GVR & RBIT_r $r$ & $\Omega \mathrm{m}$ & Ring resistivity & 10 & 34.1 & 0.09 & 0.24 & 0.43 \\
\hline GVR & RING_r & $\Omega \mathrm{m}$ & Bit resistivity & 10 & 34.1 & 0.09 & 0.24 & 0.43 \\
\hline SVWD & A_SWAV_Z & - & SVWD generic Dpoint (utility frame) & 8 & 6.8 & 0.02 & 0.05 & 0.09 \\
\hline GVR & RDBA_r & $\Omega \mathrm{m}$ & Average deep button resistivity & 10 & 34.1 & 0.09 & 0.24 & 0.43 \\
\hline PP & APRS & psi & Average annular pressure & 15 & 22.7 & 0.06 & 0.16 & 0.28 \\
\hline sonic & C_PEAK4_S & - & Four peaks with $\Delta \mathrm{T}, \mathrm{CO}$, and $\mathrm{TT}$ & 9 & 11.4 & 0.03 & 0.08 & 0.14 \\
\hline SVWD & A_SWAV_Z & - & SVWD generic Dpoint (utility frame) & 8 & 6.8 & 0.02 & 0.05 & 0.09 \\
\hline sonic & DTCO_s & $\mu \mathrm{s} / \mathrm{ft}$ & Sonic compressional $\Delta \mathrm{T}$ & 10 & 34.1 & 0.09 & 0.24 & 0.43 \\
\hline sonic & CHCO_s & - & Sonic compressional semblance & 7 & 34.1 & 0.09 & 0.24 & 0.43 \\
\hline ADN & RHOB_a & $\mathrm{g} / \mathrm{cm}^{3}$ & Average bulk density $(\rho)$ & 9 & 34.1 & 0.09 & 0.24 & 0.43 \\
\hline ADN & DRHO_a & $\mathrm{g} / \mathrm{cm}^{3}$ & Total delta density $(\rho)$ & 8 & 34.1 & 0.09 & 0.24 & 0.43 \\
\hline SVWD & A_SWAV_Z & - & SVWD generic Dpoint (utility frame) & 8 & 6.8 & 0.02 & 0.05 & 0.09 \\
\hline ADN & TNRA_a & - & Total neutron ratio & 9 & 34.1 & 0.09 & 0.24 & 0.43 \\
\hline sonic & C_PEAK4_S & - & Four peaks with $\Delta \mathrm{T}, \mathrm{CO}$, and $\mathrm{TT}$ & 9 & 11.4 & 0.03 & 0.08 & 0.14 \\
\hline ADN & ADIA_a & inch & Average hole diameter (caliper) & 8 & 34.1 & 0.09 & 0.24 & 0.43 \\
\hline SVWD & A_SWAV_Z & - & SVWD generic Dpoint (utility frame) & 8 & 6.8 & 0.02 & 0.05 & 0.09 \\
\hline
\end{tabular}

Notes: Used when the tool is slipping (no rotation). Fluid pulse telemetry frame while not rotating (slipping) $=$ update rate at $6.0 \mathrm{bps}$. ROP $=$ rate of penetration $(\mathrm{m} / \mathrm{h})$. PP $=$ PowerPulse MWD tool, GVR = geoVISION resistivity tool, SVWD = seismicVISION while drilling tool, sonic = sonicVISION while drilling tool, $A D N=$ Azimuthal Density Neutron tool (adnVISION). CO = coherence, $T T=$ traveltime. 
Table T6. Measurement performance specification of the adnVISION.

\begin{tabular}{|c|c|c|c|c|}
\hline Measurement & $\begin{array}{l}\text { Formation evaluation } \\
\text { neutron porosity } \\
\text { (pu) }\end{array}$ & $\begin{array}{l}\text { Bulk } \\
\text { density } \\
\left(\mathrm{g} / \mathrm{cm}^{3}\right)\end{array}$ & $\begin{array}{l}\text { Photoelectric } \\
\text { factor } \\
\left(\mathrm{b} / \mathrm{e}^{-}\right)\end{array}$ & $\begin{array}{l}\text { Ultrasonic } \\
\text { caliper } \\
\text { (inch) }\end{array}$ \\
\hline Range & $0-100$ & $1.0-3.05$ & $1-10$ & $1.5-3$ \\
\hline \multirow[t]{2}{*}{ Accuracy } & $\pm 0.5(<10)$ & \pm 0.015 & $\pm 5 \%$ & 0.01 \\
\hline & $\pm 0.5 \%(10-50)$ & $1.7-3.05$ & & \\
\hline Vertical resolution (inch): & 12 & 6 & 2 & 6 \\
\hline
\end{tabular}

Table T7. Measurement performance specification of the geoVISION. Vertical resolution and depth of investigation of geoVISION measurements. (See table notes.)

\begin{tabular}{|c|c|c|c|c|c|c|c|}
\hline \multirow[b]{2}{*}{ Measurement } & \multirow{2}{*}{$\begin{array}{l}\text { Vertical } \\
\text { resolution } \\
\text { (inch) }\end{array}$} & \multirow{2}{*}{$\begin{array}{l}\text { Depth of } \\
\text { investigation } \\
\text { (inch) }\end{array}$} & \multicolumn{2}{|c|}{$\begin{array}{c}\text { Diameter of investigation } \\
\left(R_{\mathrm{t}} / R_{\mathrm{xo}}\right)\end{array}$} & \multicolumn{2}{|c|}{$\begin{array}{l}\text { Horizontal resolution } \\
{\left[\left(R_{\mathrm{t}} / R_{\mathrm{xo}}=10\right) \times \pi\right] / 56}\end{array}$} & \multirow{2}{*}{$\begin{array}{l}\text { Midpoints o } \\
\text { extremes }\end{array}$} \\
\hline & & & $R_{\mathrm{t}} / R_{\mathrm{xo}}=10$ & $R_{\mathrm{t}} / R_{\mathrm{xo}}=0.1$ & $R_{\mathrm{t}} / R_{\mathrm{xo}}=10$ & $R_{\mathrm{t}} / R_{\mathrm{xo}}=0.1$ & \\
\hline Resistivity at the bit & $12-24^{*}$ & 12 & 32 & 32 & & & \\
\hline Ring resistivity & $2-3$ & 7 & 22 & 25 & & & \\
\hline \multicolumn{8}{|l|}{ Button resistivity: } \\
\hline Shallow focused & $2-3$ & 1 & 19 & 24 & 1.07 & 1.35 & 1.2 \\
\hline Medium focused & $2-3$ & 3 & 15 & 21 & 0.84 & 1.18 & 1 \\
\hline Deep focused & $2-3$ & 5 & 11 & 16 & 0.62 & 0.9 & 0.75 \\
\hline
\end{tabular}

Notes: ${ }^{*}=$ depends on bit subs. At a penetration rate of $30 \mathrm{~m} / \mathrm{h}$ and typical rotation rate (e.g., $50 \mathrm{rpm}$ ) we are oversampling vertically. $R_{\mathrm{t}}=$ true resistivity of formation. $R_{\mathrm{xo}}=$ resistivity of zone invaded by drilling fluid. Invasion may be minimal due to measurement of resistivity soon after cutting of hole. Then $R_{\mathrm{t}} / R_{\mathrm{xo}}=1$. Resistivity of our drilling fluid, seawater, is $0.19 \Omega \mathrm{m}$. Formation is $0.4-0.6 \Omega \mathrm{m}$. (e.g., below frontal thrust in 808) so $R_{\mathrm{t}} / R_{\mathrm{xo}}>1$ depending on invasion. Horizontal resolution for each of tools intermediate between extremes of $R_{\mathrm{t}} / R_{\mathrm{xo}}$ in table above. Imaging tools record 56 times with each revolution of tool. Accuracy of segment location is $\pm 1^{\circ}$. 